\title{
Stable modules and Wall's D(2)-problem
}

\author{
F. E. A. Johnson
}

\begin{abstract}
The $\mathrm{D}(2)$-problem is to determine whether for a three-dimensional complex $X$, the vanishing of 3-dimensional cohomology, in all coefficients, is enough to guarantee that $X$ is homotopically two-dimensional. We show that for finite complexes with finite fundamental group, a positive solution to the $\mathrm{D}(2)$-problem is obtained precisely when all stably free algebraic 2complexes are geometrically realizable.

The proof makes very strong use of techniques which apply to finite fundamental groups but not more generally; in particular, Yoneda's Theorem that, for finite groups, group cohomology is representable by stable modules of finite type, and also the Swan-Jacobinski Cancellation Theorem for such stable modules.
\end{abstract}

Mathematics Subject Classification (2000). Primary: 57M20, 57M05; Secondary: 20C05, 18G10, 18E25, 16E05.

Keywords. Cohomologically 2-dimensional 3-complex, algebraic 2-complex, stable module, kinvariant, geometrical realization.

\section{Introduction}

In Wall's fundamental study of Poincaré Duality [30], a finite Poincaré complex of dimension $n$ is envisaged as a cell complex of the following standard form

$$
M(\alpha)=X \cup_{\alpha} e^{n}
$$

where $X$ is a finite complex of dimension $\leq n-1$ and $\alpha: S^{n-1} \rightarrow X$ is a continuous map. This approach encounters a difficulty in dimension three, where it is unclear whether we can take $X$ to be of geometrical, rather than merely cohomological, dimension two (see also [29]). This prompts the celebrated

$\mathbf{D}(2)$ problem. Suppose that $X$ is a finite three dimensional cell complex such that $H^{3}(X ; \mathcal{B})=0$ for all local coefficient systems $\mathcal{B}$ on $X$. Is $X$ homotopy equivalent to a finite 2-dimensional complex?

The $\mathrm{D}(2)$-problem is naturally parametrized by the fundamental group of the complex involved; we say that a finitely presented group $G$ possesses the $\mathrm{D}(2)$ - 
property when every finite 3 -complex $X_{G}$ with $\pi_{1}\left(X_{G}\right) \cong G$ which is cohomologically two dimensional is indeed homotopy equivalent to a finite 2-complex. For $n \geq 3$ the corresponding $\mathrm{D}(\mathrm{n})$-problem was solved in the affirmative by Wall [30].

Our main result, the Realization Criterion below, is an interpretation, for finite groups, of the $\mathrm{D}(2)$ problem in purely two-dimensional terms, or more precisely, in terms of the homotopy types of two dimensional chain complexes over $\mathbf{Z} G$. If $G$ is a finitely presented group, by an algebraic 2-complex over $G$ we mean an exact sequence of modules and homomorphisms over $\mathbf{Z}[G]$ of the following form

$$
\mathbf{E}=\left(0 \rightarrow J \rightarrow E_{2} \rightarrow E_{1} \rightarrow E_{0} \rightarrow \mathbf{Z} \rightarrow 0\right)
$$

where $E_{r}$ is finitely generated and stably free over $\mathbf{Z}[G]$ for $0 \leq r \leq 2$. We denote by $\mathrm{Alg}_{G}$ the category whose objects are such algebraic 2-complexes, and whose morphisms are chain maps. The module $J$ is an invariant of chain homotopy equivalence and plays the role of an algebraic second homotopy group.

To any finite 2-dimensional (geometric) cell complex $K$ with $\pi_{1}(K) \cong G$ one associates functorially an object in $\mathbf{A l g}_{G}$, namely the 'cellular chain complex'

$$
C_{*}(K)=\left(0 \rightarrow \pi_{2}(K) \rightarrow C_{2}(\tilde{K}) \rightarrow C_{1}(\tilde{K}) \rightarrow C_{0}(\tilde{K}) \rightarrow \mathbf{Z} \rightarrow 0\right) ;
$$

here $\tilde{K}$ denotes the universal covering of $K$, and since $\tilde{K}$ is simply connected, we identify $H_{2}(\tilde{K} ; \mathbf{Z})$ with $\pi_{2}(\tilde{K})=\pi_{2}(K)$ by means of the Hurewicz Theorem. We reduce the problem of two -dimensional geometric homotopy type to purely algebraic terms:

Algebraicity Theorem. Let $G$ be a finitely presented group, and let $K, L$ be connected finite based 2-complexes with $\pi_{1}(K)=\pi_{1}(L)=G$; then there is a based homotopy equivalence $f: K \rightarrow L$ inducing the identity on $\pi_{1}$ if and only if $C_{*}(K)$ is chain homotopy equivalent to $C_{*}(L)$.

Let $\mathbf{E} \in \mathbf{A l g}_{G}$ be an algebraic 2-complex; by a geometric realization of $\mathbf{E}$, we mean a finite connected based 2-complex $K$ with $\pi_{1}(K)=G$ together with an algebraic chain equivalence $\varphi: C_{*}(K) \rightarrow \mathbf{E}$. A natural question is whether every algebraic 2- complex is geometrically realizable in this way; for finite fundamental groups, we show this is equivalent to the $\mathrm{D}(2)$-problem:

Realization Criterion. If $G$ is a finite group, the $\mathrm{D}(2)$-property holds for $G$ if and only each algebraic 2-complex $\mathbf{E} \in \mathbf{A l g}_{G}$ admits a geometric realization.

For finite $G$, the Realization Criterion thus gives a complete reduction of the $\mathrm{D}(2)$-problem to one involving only two dimensional data. We shall say that a finite group is 2-tame precisely when every algebraic 2-complex in $\mathbf{A l g}_{G}$ is geometrically realizable. The question of which finite groups are 2-tame is discussed in [16], [17]. By a change of emphasis, the result of Beyl, Latiolais and Waller [1], classifying geometrical homotopy types over fundamental groups of certain 3-manifolds, 
can also be interpreted in this light. In particular, cyclic groups, dihedral groups of order $4 n+2$, the binary Euclidean groups $\mathbf{T}^{*}, \mathbf{O}^{*}, \mathbf{I}^{*}$, the quaternion groups $Q(8), Q(16)$ and $Q(8 n+4)(n \geq 1)$ are all known to be 2-tame, whereas over the quaternion groups $Q\left(2^{n}\right)(n \geq 5)$, there exist algebraic 2-complexes for which no geometrical realization is as yet known. In [15] the discussion is specialised to the problem which provided the original motivation for this work, namely the theory of Poincaré 3-complexes with finite fundamental group.

One should point out that the Algebraicity Theorem stated above is stronger than the familiar statement of 'Whitehead's Theorem', namely that a based map $f: K \rightarrow L$ inducing an isomorphism on $\pi_{1}$ is a homotopy equivalence if and only if $C_{*}(f): C_{*}(K) \rightarrow C_{*}(L)$ is a chain equivalence. Here one must construct a geometrical homotopy equivalence starting from purely algebraic data. Despite the fact that it is well known, nevertheless, it does not seem to occur, stated explicitly in this form, anywhere in the literature.

One can, though not without at least a little effort, retrieve the Algebraicity Theorem in the form above either from Whitehead's general formulation of homotopy systems in [31] or the rather more specifically low-dimensional formulation given in [19]. In fact, both these formulations ('crossed modules', 'k-invariants') have slightly more structure than we need; properly speaking they refer to 'polarised homotopy', wherein isomorphisms to the higher homotopy groups, not merely $\pi_{1}$, are specified in advance. Although we shall meet k-invariants in Section 4, to avoid the unnecessary complications of a premature encounter we begin by giving a direct proof of the Algebraicity Theorem in Section 2.

In addition, we employ two specific techniques which render algebraic homotopy theory for finite fundamental groups considerably more tractable than is the case in general. The first is Yoneda's classification of module extensions [32]; the second is the detailed cancellation theory of $\mathbf{Z}[G]$-modules provided by the Swan-Jacobinski Theorem [25], [12]. In Sections 3-4, we sketch both of these in the context of the 'derived object' formulation of homological algebra.

The results of this paper also depend heavily upon the fundamental advances of several authors. In addition to those already mentioned, there are the many other works of Swan [22], [23], [24], [26], and the pioneering contributions of Cockroft and Swan [6], and Dyer and Sieradski [7], [8].

No introduction to the subject would be complete without mention of the theory of Browning, generalizing the Swan-Jacobinski Theorem to the context of chain complexes. His work, having languished in obscurity for twenty years, is at last being accorded some of the recognition which its fundamental character deserves. Browning did not publish his results, and his work is available only in the form of his thesis [2], and some difficult-to-obtain ETH pre-prints [3], [4]. In fact, we do not employ Browning's results directly, with the sole exception of a single use of a weak version of Browning's Stability Theorem in Section 6. Nevertheless, the existence of his work has informed our investigations throughout. In this context, we should also mention the extremely helpful explications of Browning's work by 
Gutierrez and Latiolais [11], and from a rather different perpective, the paper of Gruenberg [10].

The preliminary work for this paper was done between September 1996 and March 1997, an equivalent, differently stated, version of the Realization Criterion first being proved in February 1997; this version was announced at the British Topology Meeting at Oxford in April 1997. The author wishes to thank Karl Gruenberg both specifically, for donating copies of Browning's ETH preprints, and generally for many enlightening conversations over the years; and particularly Charles Thomas, whose initial suggestion it was to re-examine the problem of Poincaré 3-complexes, and whose paper [27] provided an important focus at an early stage of this work.

The paper is organised as follows: the Algebraicity Theorem is proved in Section 2; in Sections 3-4 we outline the necessary results from Swan-Jacobinski cancellation theory and Yoneda's classification theory of module extensions.

For any finite group $G$, the second homotopy group of any 2-complex $K$ with $\pi_{1}(K)=G$ determines a stable module $\Omega_{3}(\mathbf{Z})$. In Section 5 , we show that that the general question of realizing algebraic 2-complexes is reducible to the level of 'minimal' representatives of $\Omega_{3}(\mathbf{Z})$. Finally, in Section 6, we relate the discussion directly to the $\mathrm{D}(2)$ problem and prove the Realization Criterion.

\section{Reduction of 2-dimensional homotopy to algebra}

Let $G$ be a finitely presented group $G$. For $n \geq 2, \mathbf{C W}_{G}^{n}$ will denote the category whose objects are connected finite connected based cell complexes of dimension $\leq n$ such that $\pi_{1}(K)=G$. Morphisms are are based cellular maps; however, in the context in which we shall work, namely a single (based) homotopy equivalence $f: K \rightarrow L$ between given complexes $K, L$, no generality is lost by imposing the restriction that maps induce the isomorphism on $\pi_{1}$, and without further mention, we shall always assume this condition is in effect. Without loss, we assume that basepoints are 0-cells. Also, by the usual device of collapsing a maximal tree, no generality in homotopy arguments is lost by assuming all complexes are reduced, that is, possess a single 0-cell, and we shall allow ourselves this latitude without further comment.

By choosing once and for all a lifting of the basepoint to the universal covering, we associate to $K \in \mathbf{C W}_{G}^{2}$ an exact sequence $C_{*}(K)$ of $\mathbf{Z}[G]$-modules:

$$
C_{*}(K)=\left(0 \rightarrow H_{2}(\tilde{K} ; \mathbf{Z}) \rightarrow C_{2}(\tilde{K}) \rightarrow C_{1}(\tilde{K}) \rightarrow C_{0}(\tilde{K}) \rightarrow \mathbf{Z} \rightarrow 0\right) .
$$

Exactness follows from the simple connectivity of $\tilde{K}$, and by the Hurewicz Theorem we may identify $H_{2}(\tilde{K} ; \mathbf{Z})$ with $\pi_{2}(\tilde{K})=\pi_{2}(K)$. Strictly speaking, we should regard $C_{*}(K)$ as being a chain complex which is both augmented (by $C_{0}(\tilde{K}) \rightarrow$ $\mathbf{Z} \rightarrow 0$ ) and coaugmented (by $\left.0 \rightarrow H_{2}(\tilde{K} ; \mathbf{Z}) \rightarrow C_{2}(\tilde{K})\right)$. The correspondence $K \mapsto C_{*}(K)$ is functorial for based cellular maps. Thus if $\alpha: K \rightarrow L$ is a based 
cellular map there is a commutative diagram of $\mathbf{Z}[G]$-homomorphisms

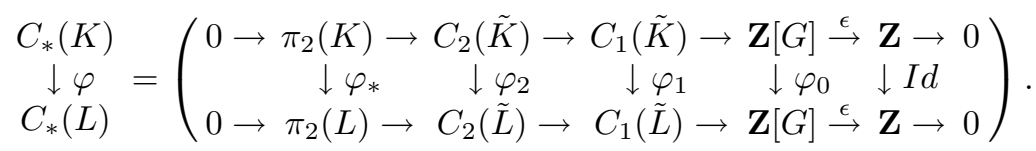

Let $\mathcal{G}=\left\langle x_{1}, \cdots, x_{g} \mid W_{1}, \cdots, W_{r}\right\rangle$ be a finite presentation for $G$, and let $y=$ $y\left(x_{1}, \cdots, x_{g}\right)$ be a word in the generators. Consider the presentation

$$
\mathcal{G}(y)=\left\langle x_{1}, \cdots, x_{g+1} \mid W_{1}, \cdots, W_{r+1}\right\rangle
$$

where $W_{r+1}=x_{g+1}^{-1} y$. Then $\mathcal{G}(y)$ is also a presentation of $G$; the passage from $\mathcal{G}$ to $\mathcal{G}(y)$ is effected by a so-called Tietze transformation of the first kind. If $K=\mathcal{K}_{\mathcal{G}}$ is the Cayley complex of $\mathcal{G}$, we denote by $K(y)$ the Cayley complex of $\mathcal{G}(y) ; K(y)$ is (simply) homotopy equivalent to $K$.

Proposition 2.1. Let $K, L$ be finite reduced 2-complexes with $\pi_{1}(K)=\pi_{1}(L)=$ $G$. Then there exist finite reduced 2 -complexes $K_{1}, L_{1}$ such that

(i) $K_{1} \simeq K$ and $L_{1} \simeq L$;

(ii) $K_{1}^{(1)} \equiv L_{1}^{(1)}$.

Proof. Let $\left\{x_{1}, \cdots, x_{m}\right\}$ (resp. $\left\{y_{1}, \cdots, y_{n}\right\}$ ) be the generating sets of $G$ corresponding to the 1 -cells of $K$ (resp. $L$ ), and regard $y_{i}$ as a word in $\left\{x_{1}, \cdots, x_{m}\right\}$. We can regard $K$ as the Cayley complex of some presentation. Starting with the generating set $\left\{x_{1}, \cdots, x_{m}\right\}$ and adding the elements $y_{1}, \cdots, y_{n}$ successively, we obtain a sequence of presentations

$$
\mathcal{G}, \mathcal{G}\left(y_{1}\right), \mathcal{G}\left(y_{1}, y_{2}\right), \cdots \cdots, \mathcal{G}\left(y_{1}, y_{2}, \cdots, y_{n}\right)
$$

and a sequence of (simple) homotopy equivalences

$$
K \subset K\left(y_{1}\right) \subset K\left(y_{1}, y_{2}\right) \subset \cdots \cdots \subset K\left(y_{1}, y_{2}, \cdots, y_{n}\right)=K_{1} .
$$

Likewise, regarding $L$ as the Cayley complex $\mathcal{L}=\mathcal{L}_{\mathcal{H}}$ of some presentation, and adding the elements $x_{1}, \cdots, x_{m}$ successively, starting with the generating set $\left\{y_{1}, \cdots, y_{n}\right\}$, we obtain a sequence of presentations

$$
\mathcal{H}, \mathcal{H}\left(x_{1}\right), \mathcal{H}\left(x_{1}, x_{2}\right), \cdots \cdots, \mathcal{H}\left(x_{1}, x_{2}, \cdots, x_{m}\right),
$$

and a sequence of (simple) homotopy equivalences

$$
L \subset L\left(x_{1}\right) \subset L\left(x_{1}, x_{2}\right) \subset \cdots \cdots \subset L\left(x_{1}, x_{2}, \cdots, x_{m}\right)=L_{1} .
$$

Then in some ordering, the 1-cells of $K_{1}$ and those of $L_{1}$ correspond to the generating elements $\left(x_{1}, x_{2}, \cdots, x_{m}, y_{1}, y_{2}, \cdots, y_{n}\right)$; that is, we may identify $K_{1}^{(1)}$ with $L_{1}^{(1)}$

Proposition 2.2. Let $K, L$ be finite reduced 2-complexes with $\pi_{1}(K)=\pi_{1}(L)=$ $G$, and suppose that $K^{(1)}=L^{(1)}$. If $\nu: C_{*}(\tilde{K}) \rightarrow C_{*}(\tilde{L})$ is an augmented $\mathbf{Z}[G]$ chain map, then $\nu$ is chain homotopic to a chain map $\mu$ which restricts to the identity on $C_{1}(\tilde{K})=C_{1}(\tilde{L})$. 
Proof. Begin with the chain map

$$
\begin{gathered}
C_{*}(\tilde{K}) \\
\downarrow v \\
C_{*}(\tilde{L})
\end{gathered}=\left(\begin{array}{cccc}
C_{2}(\tilde{K}) \stackrel{\partial_{2}}{\rightarrow} C_{1}(\tilde{K}) \stackrel{\partial_{1}}{\rightarrow} \mathbf{Z}[G] \stackrel{\epsilon}{\rightarrow} \mathbf{Z} \rightarrow 0 \\
\downarrow \nu_{2} & \downarrow \nu_{1} & \downarrow \nu_{0} & \downarrow I d \\
C_{2}(\tilde{L}) \stackrel{\delta_{2}}{\rightarrow} & C_{1}(\tilde{L}) \stackrel{\partial_{1}}{\rightarrow} \mathbf{Z}[G] \stackrel{\epsilon}{\rightarrow} \mathbf{Z} \rightarrow 0
\end{array}\right)
$$

where, by hypothesis, $C_{1}(\tilde{L})=C_{1}(\tilde{K})$ and $\partial_{1}^{K}=\partial_{1}^{L}=\partial_{1}$. Then $\operatorname{Im}\left(I d-\nu_{0}\right) \subset$ $\operatorname{Ker}(\epsilon)=\operatorname{Im}\left(\partial_{1}\right)$. Choose a $\mathbf{Z}[G]$-homomorphism $h_{0}: \mathbf{Z}[G] \rightarrow C_{1}(\tilde{L})$ such that $\partial_{1} h_{0}=I d-\nu_{0}$, and put $\lambda_{1}=h_{0} \partial_{1}+\nu_{1}, \lambda_{2}=\nu_{2}$. We obtain a chain map $\lambda: C_{*}(\tilde{K}) \rightarrow C_{*}(\tilde{L})$

$$
\begin{gathered}
C_{*}(\tilde{K}) \\
\downarrow \lambda \\
C_{*}(\tilde{L})
\end{gathered}=\left(\begin{array}{cccc}
C_{2}(\tilde{K}) \stackrel{\partial_{2}}{\rightarrow} & C_{1}(\tilde{K}) \stackrel{\partial_{1}}{\rightarrow} \mathbf{Z}[G] \stackrel{\epsilon}{\rightarrow} \mathbf{Z} \rightarrow 0 \\
\downarrow \lambda_{2} & \downarrow \lambda_{1} & \downarrow I d & \downarrow I d \\
C_{2}(\tilde{L}) \stackrel{\delta_{2}}{\rightarrow} & C_{1}(\tilde{L}) \stackrel{\partial_{1}}{\rightarrow} \mathbf{Z}[G] \stackrel{\epsilon}{\rightarrow} \mathbf{Z} \rightarrow 0 .
\end{array}\right)
$$

Clearly $\operatorname{Im}\left(I d_{1}-\lambda_{1}\right) \subset \operatorname{Ker}\left(\partial_{1}\right)=\operatorname{Im}\left(\delta_{2}\right)$. Choose a $\mathbf{Z}[G]$-homomorphism $h_{1}$ : $C_{1}(\tilde{K}) \rightarrow C_{1}(\tilde{K})$ such that $\delta_{2} h_{1}=I d_{1}-\lambda_{1}$. Put $\mu_{2}=h_{1} \partial_{2}+\lambda_{2}$. Then

$$
\begin{array}{rlc}
\delta_{2} \mu_{2} & = & \delta_{2}\left(h_{1} \partial_{2}+\lambda_{2}\right) \\
& = & \delta_{2} h_{1} \partial_{2}+\delta_{2} \lambda_{2} \\
& = & \partial_{2}-\lambda_{1} \partial_{2}+\delta_{2} \lambda_{2} \\
& = & \partial_{2} .
\end{array}
$$

The following commutative diagram defines the desired chain map $\mu$

$$
\begin{gathered}
C_{2}(\tilde{K}) \stackrel{\partial_{2}}{\rightarrow} C_{1}(\tilde{K}) \stackrel{\partial_{1}}{\rightarrow} \mathbf{Z}[G] \stackrel{\epsilon}{\rightarrow} \mathbf{Z} \rightarrow 0 \\
\downarrow \mu_{2} \rightarrow \downarrow d d \quad \downarrow I d \quad \downarrow I d \\
C_{2}(\tilde{L}) \stackrel{\delta_{2}}{\rightarrow} C_{1}(\tilde{L}) \stackrel{\partial_{1}}{\rightarrow} \mathbf{Z}[G] \stackrel{\epsilon}{\rightarrow} \mathbf{Z} \rightarrow 0,
\end{gathered}
$$

and $h=\left(h_{1}, h_{0}\right)$ is a chain homotopy from $\mu$ to $\nu$.

If $\varphi: C_{*}(K) \rightarrow C_{*}(L)$ is an algebraic chain map, by a realization of $\varphi$ we mean a cellular map $f: K \rightarrow L$ such that $C_{*}(f)=\varphi$. The following Lemma, gives conditions under which some chain maps are realizable. It is modelled on the proof of result of Gutierrez and Latiolais ([11] Prop. (2.3)) in a a slightly more general context, amd is included here for the sake of completeness.

Lemma 2.3. Let $G$ be a finitely presented group, and let $K, L$ be finite reduced 2-dimensional cell complexes such that $\pi_{1}(K)=\pi(L)=G$, and $K^{(1)} \equiv L^{(1)}$; if $\varphi: C_{*}(K) \rightarrow C_{*}(L)$ is an algebraic chain map such that $\varphi_{\mid C_{i}(K)}=\operatorname{Id}$ then there exists a cellular map $f: K \rightarrow L$ such that

(i) $f$ extends the identity of $K^{(1)} \equiv L^{(1)}$ and induces the identity on $\pi_{1}$, and

(ii) $C_{*}(f)=\varphi$. 
Proof. By hypothesis, the following diagram commutes:

$$
\begin{array}{ccc}
C_{2}(K) & \stackrel{\partial_{2}^{K}}{\rightarrow} & C_{1}(K) \\
\downarrow \varphi_{2} & & \| \\
C_{2}(L) & \stackrel{\partial_{2}^{L}}{\rightarrow} & C_{1}(L) .
\end{array}
$$

Begin by choosing some cellular map $\lambda: K \rightarrow L$ with the property that $\lambda$ extends the identity of $K^{(1)} \equiv L^{(1)}$ and induces the identity on $\pi_{1}$. For example, we may first attach cells of dimension $\geq 3$ to $L$ to kill homotopy groups in dimensions $\geq 3$, so forming a $K(G, 1)$ space with $K(G, 1)^{(2)}=L$. The identity map on $K^{(1)}$ then extends to a map $\lambda: K \rightarrow K(G, 1)$ which classifies the universal $G$-bundle $\tilde{K} \rightarrow K$ and which induces the identity on $\pi_{1}$. Without loss of generality, the cellular approximation theorem allows us to choose $\lambda$ to be cellular, extending the identity on $K^{(1)}$. In particular $\lambda: K \rightarrow \lambda(K) \subset K(G, 1)^{(2)}=L$.

The chain map $C_{*}(\lambda)$ induced by $\lambda$ makes the following diagram commute:

$$
\begin{array}{ccc}
C_{2}(K) & \stackrel{\partial_{2}^{K}}{\rightarrow} & C_{1}(K) \\
\downarrow \lambda_{2} & & \| \\
C_{2}(L) & \stackrel{\partial_{2}^{L}}{\rightarrow} & C_{1}(L) .
\end{array}
$$

In particular, $\partial_{2}^{L}\left(\varphi_{2}-\lambda_{2}\right)=0$, and since the Hurewicz Theorem allows us to identify $\operatorname{Ker}\left(\partial_{2}^{L}\right)=H_{2}(\tilde{L} ; \mathbf{Z})$ can be identified with $\pi_{2}(L)$, then $\varphi_{2}-\lambda_{2}$ gives a $\mathbf{Z}[G]$-homomorphism

$$
\varphi_{2}-\lambda_{2}: C_{2}(K) \rightarrow \pi_{2}(L) .
$$

Let $\left\{E_{1}, \cdots, E_{r}\right\}$ denote the 2-cells and let $\left\{\hat{E}_{1}, \cdots, \hat{E}_{r}\right\}$ denote the $\mathbf{Z}[G]$-basis of $C_{2}(K)$ obtained by to $\tilde{K}$; let $h_{i}: S^{2} \rightarrow L$ be a map whose homotopy class $\left[h_{i}\right]$ satisfies

$$
\left[h_{i}\right]=\left(\varphi_{2}-\lambda_{2}\right)\left(\hat{E}_{i}\right)
$$

Let $c_{i}: E_{i} \rightarrow E_{i} \vee S_{i}^{2}$ denote a 'pinch map' obtained by identifying an interior circle in $E_{i}$ to a point. We may write

$$
K=\left(\coprod_{i} E_{i}\right) / \sim
$$

where ' $\sim$ ' makes identifications in the boundaries of the $E_{i}$. Put

$$
X=\left(\coprod_{i} E_{i} \vee S_{i}^{2}\right) / \sim
$$

where ' $\sim$ ' makes the same identifications as before, observing that $E_{i}$ and $E_{i} \vee S_{i}^{2}$ have the same boundary. One may think of $X$ as formed from $K$ by attaching a copy of $S^{2}$ at a single interior point within each 2-cell of $K$. Put $\lambda_{i}=\lambda_{\mid E_{i}}$ and define

$$
c=\left(\coprod_{i} c_{i}\right) / \sim: K \rightarrow X \quad ; \quad \Psi=\left(\coprod_{i} \lambda_{i} \vee h_{i}\right) / \sim: X \rightarrow L
$$


and put $f=\Psi \circ c: K \rightarrow L$. $f$ is a basepoint preserving map ; it is straightforward to see that $f$ extends the identity on $K^{(1)}$ and induces the identity on $\pi_{1}$. If $f_{n}: C_{n}(K) \rightarrow C_{n}(L)$ denotes the induced map on $n$-chains, one calculates easily that

$$
f_{2}\left(\hat{E}_{i}\right)=\lambda_{2}\left(\hat{E}_{i}\right)+\left[h_{i}\right]
$$

where we identify $\left[h_{i}\right] \in \pi_{2}(L)$ with its image in $C_{2}(L)$. However, by choice, $\left(\varphi_{2}-\lambda_{2}\right)\left(\hat{E}_{i}\right)=\left[h_{i}\right]$. Thus

$$
\begin{aligned}
f_{2}\left(\hat{E}_{i}\right) & =\lambda_{2}\left(\hat{E}_{i}\right)+\left(\varphi_{2}-\lambda_{2}\right)\left(\hat{E}_{i}\right) \\
& =\varphi_{2}\left(\hat{E}_{i}\right)
\end{aligned}
$$

and $f_{2}=\varphi_{2}$ as required.

Proposition 2.4. Let $K, L$ be finite reduced 2-complexes with $\pi_{1}(K)=\pi_{1}(L)=$ $G$, and suppose that $\varphi: C_{*}(\tilde{K}) \rightarrow C_{*}(\tilde{L})$ is an algebraic chain homotopy equivalence; then there exists a cellular homotopy equivalence $f: K \rightarrow L$ whose induced map on the fundamental group is the identity.

Proof. By (2.1), we may, without loss of generality, assume that $K^{(1)}=L^{(1)}$. Now $H_{0}(\tilde{K} ; \mathbf{Z})$ and $H_{0}(\tilde{L} ; \mathbf{Z})$ are canonically isomorphic to $\mathbf{Z}$, so that the isomorphism $\varphi_{*}: H_{0}(\tilde{K} ; \mathbf{Z}) \rightarrow H_{0}(\tilde{L} ; \mathbf{Z})$ is \pm Id. By replacing $\varphi$ by $-\varphi$, if necessary, we may suppose that $\varphi$ induces an isomorphism on $H_{0}$.

Now by (2.2), we may further assume that $\mu$ restricts to the identity on $C_{1}(\tilde{K})=C_{1}(\tilde{L})$. Finally, applying $(2.3)$, we obtain a cellular map $f: K \rightarrow L$ inducing the identity on the fundamental group and such that $f_{*}=\mu: H_{2}(\tilde{K} ; \mathbf{Z}) \rightarrow$ $H_{2}(\tilde{L} ; \mathbf{Z})$. In particular, $f_{*}: H_{2}(\tilde{K} ; \mathbf{Z}) \rightarrow H_{2}(\tilde{L} ; \mathbf{Z})$ is an isomorphism, so that $f$ is a homotopy equivalence, by Whitehead's Theorem.

As a corollary, we have:

Algebraicity Theorem. Let $G$ be a finitely presented group, and let $K, L$ be connected finite based 2-complexes with $\pi_{1}(K)=\pi_{1}(L)=G$; then $K$ is homotopy equivalent to $L$ if and only if $C_{*}(K)$ is chain homotopy equivalent to $C_{*}(L)$.

\section{Stable modules and derived functors}

Throughout rest of the paper, $G$ will denote a finite group, although at the more important points we shall re-emphasize the fact; we denote by $\mathcal{F}(\mathbf{Z}[G])$ the category of $\mathbf{Z}[G]$ lattices, that is, finitely generated (right) $\mathbf{Z}[G]$-modules whose underlying $\mathbf{Z}$-modules are free. We let ' $\sim$ ' denote the stability relation on $\mathcal{F}(\mathbf{Z}[G])$; that is

$$
M_{1} \sim M_{2} \Longleftrightarrow M_{1} \oplus \mathbf{Z}[G]^{n_{1}} \cong M_{2} \oplus \mathbf{Z}[G]^{n_{2}}
$$


for some $n_{1}, n_{2} \geq 0$, and put

$$
\operatorname{Stab}(\mathbf{Z}[G])=\mathcal{F}(\mathbf{Z}[G]) / \sim .
$$

For any $\mathbf{Z}[G]$-lattice $M$, we denote by $[M] \in \operatorname{Stab}(\mathbf{Z}[G])$ the class of $M$ under $\sim$. Recall Swan's generalization of 'Schanuel's Lemma' [22]; if

$$
0 \rightarrow B \rightarrow F_{n} \rightarrow \cdots \rightarrow F_{0} \rightarrow A \rightarrow 0,
$$

and

$$
0 \rightarrow B^{\prime} \rightarrow F_{n}^{\prime} \rightarrow \cdots \rightarrow F_{0}^{\prime} \rightarrow A^{\prime} \rightarrow 0
$$

are exact sequences of $\mathbf{Z}[G]$-modules in which $F_{i}, F_{j}^{\prime}$ are finitely generated and free, then $A \cong A^{\prime}$ implies that $B \oplus F \cong B^{\prime} \oplus F^{\prime}$ for some finitely generated free $\mathbf{Z}[G]$-modules $F, F^{\prime}$. To each module $M \in \mathcal{F}(\mathbf{Z}[G])$, we can associate a sequence $\left(\Omega_{r}\right)_{r \geq 0}$ of stable modules defined by the condition that $\Omega_{n}(M)$ is the stable class $[D]$ of any module $D \in \mathcal{F}(\mathbf{Z}[G])$ for which there exists an exact sequence of the form

$$
0 \rightarrow D \rightarrow F_{n-1} \rightarrow \cdots \rightarrow F_{0} \rightarrow M \rightarrow 0
$$

where each $F_{r}$ is a finitely generated free module over $\mathbf{Z}[G]$. Likewise, to each module $M \in \mathcal{F}(\mathbf{Z}[G])$, we associate a sequence $\left(\Omega_{-r}(M)\right)_{r \geq 0}$ of stable modules defined by the condition that $\Omega_{-n}(M)$ is the stable class $[D]$ of any module $D \in$ $\mathcal{F}(\mathbf{Z}[G])$ for which there exists an exact sequence of the form

$$
0 \rightarrow M \rightarrow F_{0} \rightarrow \cdots \rightarrow F_{n-1} \rightarrow D \rightarrow 0
$$

where each $F_{r}$ is a finitely generated free module over $\mathbf{Z}[G]$.

We introduce duality into $\mathcal{F}(\mathbf{Z}[G])$ as follows; when $M \in \mathcal{F}(\mathbf{Z}[G])$ we denote by $M^{*}$ the right $\mathbf{Z}[G]$-module whose underlying $\mathbf{Z}$-module is $\operatorname{Hom}_{\mathbf{Z}}(M, \mathbf{Z})$ and whose $G$-action is given by

$$
(f \bullet g)(m)=f\left(m g^{-1}\right) .
$$

Under our hypothesis that $G$ is finite, $M^{*}$ is a model for the $\mathbf{Z}[G]$-dual of $M$. This is a special case of the 'Eckmann-Shapiro Lemma'. As an explicit model for $\operatorname{Hom}_{\mathbf{Z}[G]}(M, \mathbf{Z}[G])$ we may take sequences $\alpha=\left(\alpha_{x}\right)_{x \in G}$, with each $\alpha_{x} \in$ $\operatorname{Hom}_{\mathbf{Z}}(M, \mathbf{Z})$, satisfying the condition $\alpha_{g h}(m)=\alpha_{h}\left(m g^{-1}\right)$. In this case, the right $G$-action is given by $(\alpha \bullet x)_{g}=\alpha_{x g}$, and the mapping

$$
L: \operatorname{Hom}_{\mathbf{Z}[G]}(M, \mathbf{Z}[G]) \rightarrow M^{*} ; \quad L\left(\left(\alpha_{g}\right)_{g \in G}\right)=\alpha_{1}
$$

is an isomorphism. ${ }^{\dagger}$ There is now a natural equivalence $\nu: M \rightarrow M^{* *}$ given by $\nu(x)(\alpha)=\alpha(x)$. Duality is introduced into $\operatorname{Stab}(\mathbf{Z}[G])$ by writing $[M]^{*}=\left[M^{*}\right]$.

Proposition 3.1. For any module $M \in \mathcal{F}(\mathbf{Z}[G])$ we have the following relations:

$$
\begin{array}{lll}
\Omega_{m}\left(\Omega_{n}(M)\right) & =\Omega_{m+n}(M) ; \\
\Omega_{n}\left(M^{*}\right) & = & \Omega_{-n}(M)^{*} .
\end{array}
$$

$\dagger$ The referee emphasizes the point that this ceases to be true if the hypothesis that $G$ be finite is dropped. A good example is obtained by taking $M=\mathbf{Z}$, the trivial module over the infinite cyclic group $\mathbf{T}$. Then $M^{*} \cong M$ but $\operatorname{Hom}_{\mathbf{Z}[\mathbf{T}]}(M, \mathbf{Z}[\mathbf{T}])=0$. 
The trivial module $\mathbf{Z}$ is self-dual over $\mathbf{Z}[G]$, so that:

Proposition 3.2. For any finite group $G$,

(i) $\quad \Omega_{1}(\mathbf{Z}) \quad=[\mathbf{I}(G)]$;

(ii) $\quad \Omega_{-1}(\mathbf{Z}) \quad=\left[\mathbf{I}(G)^{*}\right]$.

We wish to interpret the constructions $\Omega_{k}$ in terms of derived categories. This is essentially well known. In a different, though parallel, context, there is an elegant exposition in the monograph of Carlson [5]. A systematic treatment of group cohomology in the exact context required for this paper can be found in $[14]$.

Let $f, g: M \rightarrow N$ be $\mathbf{Z}[G]$-homomorphisms where $M, N \in \mathcal{F}(\mathbf{Z}[G])$. We write $f \approx g$ when $f-g$ factors through a projective module; that is, when $f-g$ can be written as a composite $f-g=\beta \circ \alpha$, of $\mathbf{Z}[G]$-homomorphisms $\alpha: M \rightarrow P$ and $\beta: P \rightarrow N$. where $P$ is projective over $\mathbf{Z}[G]$. It is straightforward to check that $\approx$ is an equivalence relation compatible with addition and two sided composition. By the derived category $\operatorname{Der}(\mathcal{F}(\mathbf{Z}[G]))$ of $\mathcal{F}(\mathbf{Z}[G])$, we mean the category whose objects are modules in $\mathcal{F}(\mathbf{Z}[G])$, and in which, for any two modules $M, N$, the set of morphisms $\operatorname{Hom}_{\mathcal{D} \text { er }}(M, N)$ is given by

$$
\operatorname{Hom}_{\mathcal{D e r}}(M, N)=\operatorname{Hom}_{\mathbf{Z}[G]}(M, N) / \approx .
$$

Let $M_{1}, M_{2} \in \mathcal{F}(\mathbf{Z}[G])$; it is clear that if $M_{1} \oplus \mathbf{Z}[G]^{m_{1}} \cong M_{2} \oplus \mathbf{Z}[G]^{m_{1}}$ then $M_{1}$ and $M_{2}$ are isomorphic in the derived category. Thus each stable module $\Omega_{k}(M)$ gives rise to an isomorphism class, denoted by the same symbol, in the derived category. The traditional interpretation of $H^{k}(-,-)$ as the $k^{t h}$ derived functor of $\operatorname{Hom}(-,-)$ can be made explicit in this context (see [13]).

Proposition 3.3. On the category $\mathcal{F}(\mathbf{Z}[G])$, for all $k \geq 1$ there is a natural equivalence of functors $\nu: H^{k}(M, N) \rightarrow \operatorname{Hom}_{\mathcal{D e r}}\left(\Omega_{k}(M), N\right)$.

In particular, we see that, in the derived category, $\Omega_{k}(M)$ is a corepresenting object for the functor $X \mapsto H^{k}(M, X)$. Moreover, this statement remains true for $k=0$ provided $H^{0}(M, N)$ is 'correctly interpreted' as Tate cohomology. We note that, $\Omega_{1}(\mathbf{Z})$ is the stable class of the augmentation ideal, and $\Omega_{2}(\mathbf{Z})$ is the stable class of the so-called 'second Fox ideal' of a presentation. Since the category $\mathcal{F}(\mathbf{Z}[G])$ has good duality properties, one sees also that:

Proposition 3.4. On the category $\mathcal{F}(\mathbf{Z}[G])$ there are natural equivalences

$$
\nu: H^{k}(M, N) \rightarrow \operatorname{Hom}_{\mathcal{D e r}}\left(M, \Omega_{-k}(N)\right)
$$

all $k \geq 1$; that is, in the derived category, $\Omega_{-k}(N)$ is a representing object for the functor $X \mapsto H^{k}(X, N)$. 
If $M \in \mathcal{F}(\mathbf{Z}[G])$, we denote by $[M]$ the stable module consisting of all modules in $\mathcal{F}(\mathbf{Z}[G])$ which are stably equivalent to $M$. Then $[M]$ has a natural combinatorial structure, namely that of a directed graph in which the vertices are the modules $N \in[M]$, and where we draw an arrow $N \rightarrow N \oplus \mathbf{Z}[G]$. It is clear that there are no loops in the corresponding undirected graph, so that $[M]$ is a tree. As we shall see, the structure is rather more specific. A module $C \in \mathcal{F}(\mathbf{Z}[G])$ is said to have the cancellation property when, for any $N \in \mathcal{F}(\mathbf{Z}[G])$, an isomorphism of the form

$$
N \oplus \mathbf{Z}[G]^{c} \cong C \oplus \mathbf{Z}[G]^{n} \Longrightarrow N \cong C \oplus \mathbf{Z}[G]^{n-c}
$$

provided $c \leq n$. We say that the finite group $G$ has the cancellation property for free modules when finitely generated stably free modules over $\mathbf{Z}[G]$ are actually free; that is, when $B \oplus \mathbf{Z}[G]^{\beta} \cong \mathbf{Z}[G]^{\alpha}$ implies that $B \cong \mathbf{Z}[G]^{\alpha-\beta}$.

Recall that if $C \in \mathcal{F}(\mathbf{Z}[G])$, we say that $C$ satisfies the Eichler condition when, in the Wedderburn decomposition of $\operatorname{End}_{\mathbf{Q}[G]}(C \otimes \mathbf{Q})$, no simple factor is a totally definite quaternion algebra. The following theorem of Swan ([25] Chap. 9), building on Jacobinski's Cancellation Theorem [12], gives a sufficient condition for a module in $\mathcal{F}(\mathbf{Z}[G])$ to possess the cancellation property.

Theorem 3.5. Let $G$ be a finite group, and let $M \in \mathcal{F}(\mathbf{Z}[G])$. If $M \oplus \mathbf{Z}[G]$ satisfies the Eichler condition, then $M \oplus \mathbf{Z}[G]$ has the cancellation property.

By a fork, we mean a locally finite tree of the following type (Fig. 1) with a single vertex at each positive integral height, and a finite set of vertices at height zero, so that there is no branching above level 1 .

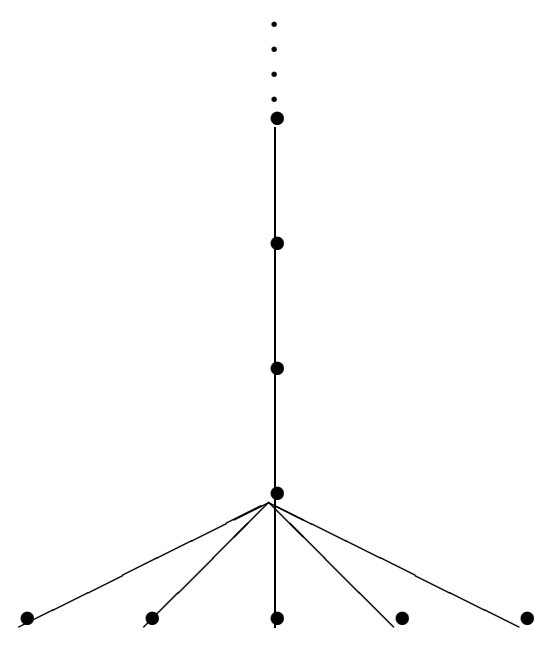

Figure 1

It is a direct consequence of the Swan-Jacobinski Theorem that if $M \in \mathcal{F}(\mathbf{Z}[G])$ is rationally equivalent to the augmentation ideal $\mathbf{I}(G)$, then any $N \in[M]$ of non- 
minimal rank has the cancellation property. It follows easily that:

Proposition 3.6. $\Omega_{2 n+1}(\mathbf{Z})$ is a fork for each $n \geq 1$.

\section{Module extensions}

We begin by summarizing Yoneda's cohomological classification of module extensions [32]; in fact, we shall need a relative version of Yoneda's extension theory, where all exact sequences are taken over the category $\mathcal{F}(\mathbf{Z}[G])$. This is also well known in principle, but it is difficult to find an account in the literature which covers our requirements exactly. In fact, the precise details required here may be found in [14]. Thus let $M, N$ be modules in $\mathcal{F}(\mathbf{Z}[G])$; then $\mathbf{E x t}_{\mathcal{F}(\mathbf{Z}[G])}^{n}(M, N)$ will denote the category whose objects are exact sequences of $\mathbf{Z}[G]$-modules and homomorphisms

$$
\mathbf{E}=\left(0 \rightarrow N \rightarrow E_{n-1} \rightarrow \cdots \cdots \rightarrow E_{0} \rightarrow M \rightarrow 0\right)
$$

in which all modules lie in $\mathcal{F}(\mathbf{Z}[G])$, and whose morphisms are commutative diagrams of $\mathbf{Z}[G]$-homomorphisms thus:

$$
\begin{aligned}
& \mathbf{E} \\
& \downarrow h \\
& \mathbf{F}
\end{aligned}=\left(\begin{array}{cccc}
0 \rightarrow & N \rightarrow E_{n-1} \rightarrow \cdots \cdots \rightarrow E_{0} \rightarrow M \rightarrow 0 \\
& \downarrow h_{+} \downarrow h_{n-1} & & \downarrow h_{0} \downarrow h_{-} \\
0 \rightarrow & N \rightarrow F_{n-1} \rightarrow \cdots \cdots \rightarrow F_{0} \rightarrow M \rightarrow 0
\end{array}\right) .
$$

When $G$ is understood, we abbreviate $\operatorname{Ext}_{\mathcal{F}(\mathbf{Z}[G])}^{n}(M, N)$ to $\mathbf{E x t}^{n}(M, N)$.

If $\mathbf{E}, \mathbf{F} \in \mathbf{E x t}^{n}(A, B)$, a morphism $\varphi: E \stackrel{\rightarrow}{\rightarrow}$ is said to be an elementary congruence when it induces the identity at both ends thus:

$$
\begin{aligned}
& \mathbf{E} \\
& \downarrow \varphi \\
& \mathbf{F}
\end{aligned}=\left(\begin{array}{cccc}
0 \rightarrow B \rightarrow E_{n-1} \rightarrow \cdots \cdots & E_{0} \rightarrow A \rightarrow 0 \\
& \downarrow \text { Id } \downarrow \varphi_{n-1} & \downarrow \varphi_{0} & \downarrow \text { Id } \\
0 \rightarrow B \rightarrow F_{n-1} \rightarrow \cdots \cdots \rightarrow F_{0} \rightarrow A \rightarrow 0
\end{array}\right) .
$$

We write $\mathbf{E} \rightsquigarrow \mathbf{F}$ when there exists an elementary congruence $\varphi: \mathbf{E} \rightarrow \mathbf{F}$. In the case $n=1$, it is a consequence of the Five Lemma that elementary congruence is an equivalence relation on $\mathbf{E x t}{ }^{1}(M, N)$, and we write:

$$
\operatorname{Ext}^{1}(M, N)=\operatorname{Ext}^{1}(M, N) / \rightsquigarrow .
$$

By contrast, $\rightsquigarrow$ is not an equivalence relation when $n \geq 2$; in this case, we denote by ' $\equiv$ ' the equivalence relation generated by ' $\rightsquigarrow$ '; that is $\mathbf{E}, \mathbf{F} \in \mathbf{E x t}^{n}(M, N)$ are said to be congruent, written $\mathbf{E} \equiv \mathbf{F}$, when there exists a sequence $\mathbf{E}_{\mathbf{r}},(0 \leq r \leq$ $m) \in \mathbf{E x t}^{n}(M, N)$ such that $\mathbf{E}=\mathbf{E}_{\mathbf{0}}, \mathbf{F}=E_{m}$, and for each $r \leq m-1$, either $\mathbf{E}_{\mathbf{r}} \rightsquigarrow \mathbf{E}_{\mathbf{r}+\mathbf{1}}$ or $\mathbf{E}_{\mathbf{r}+\mathbf{1}} \rightsquigarrow \mathbf{E}_{\mathbf{r}}$. We denote by $\operatorname{Ext}^{n}(M, N)$ the quotient set

$$
\operatorname{Ext}^{n}(M, N)=\mathbf{E x t}^{n}(M, N) / \equiv .
$$

Suppose that $K \in \Omega_{n}(M)$, and fix an extension

$$
\mathbf{F}=\left(0 \rightarrow K \rightarrow F_{n-1} \rightarrow \cdots \cdots \rightarrow F_{0} \rightarrow M \rightarrow 0\right)
$$


where each $F_{i}$ is finitely generated free $\mathbf{Z}[G]$-module. Suppose that

$$
\mathbf{E}=\left(0 \rightarrow N \rightarrow E_{n-1} \rightarrow \cdots \cdots \rightarrow E_{0} \rightarrow M \rightarrow 0\right) \in \mathbf{E x t}^{n}(M, N)
$$

The universal property of free modules gives a commutative diagram of $\mathbf{Z}[G]$ modules thus:

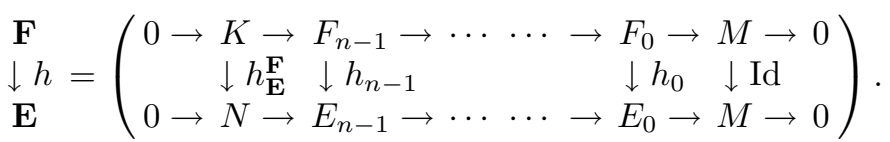

The essence of Yoneda's Theorem [32], [14] is that, for fixed F, the correspondence $\mathbf{E} \mapsto h_{\mathbf{E}}^{\mathbf{F}} \in \operatorname{Hom}_{\mathbf{Z}[G]}(K, N)$ gives rise to a bijection

$$
\operatorname{Ext}^{n}(M, N) \stackrel{\simeq}{\longrightarrow} \operatorname{Hom}_{\mathcal{D e r}}(K, N) \cong \operatorname{Hom}_{\mathcal{D e r}}\left(\Omega_{n}(M), N\right) \text {. }
$$

Using the corepresentability result of (3.3), we obtain the following version of Yoneda's Theorem:

Proposition 4.1. For each $M \in \mathcal{F}(\mathbf{Z}[G])$, the functors $N \mapsto \operatorname{Ext}^{n}(M, N)$ and $N \mapsto H^{n}(M, N)$ are naturally equivalent for all $n \geq 1$.

This bijection imposes a (slightly unnatural) group structure on $\operatorname{Ext}^{n}(M, N)$. We note however, that in the case $n=1$, the group structure is entirely natural, being given by Baer sum ([18] Chap. 3), and in that case the Yoneda map is an equivalence of group valued functors.

For any $M, N \in \mathcal{F}(\mathbf{Z}[G])$ we denote by $\operatorname{Free}^{n}(M, N)$ the subclass of $\mathbf{E x t}^{n}(M, N)$ consisting of extensions of the form

$$
0 \rightarrow N \rightarrow A_{n-1} \rightarrow \cdots \cdots \rightarrow A_{1} \rightarrow A_{0} \rightarrow M \rightarrow 0
$$

with $A_{i} \in \mathcal{F}(\mathbf{Z}[G])$ in which each $A_{i}$ is free. Likewise $\operatorname{Proj}^{n}(M, N)$ (resp. $\left.\mathbf{S F}^{n}(M, N)\right)$ will denote the subclass of $\mathbf{E x t}^{n}(M, N)$ which each $A_{i}$ is projective (resp. stably free). We have inclusions

$$
\operatorname{Free}^{n}(M, N) \subset \mathbf{S F}^{n}(M, N) \subset \operatorname{Proj}^{n}(M, N) \subset \mathbf{E x t}^{n}(M, N) .
$$

Proposition 4.2. Let $M, N \in \mathcal{F}(\mathbf{Z}[G])$; then for each $n \geq 1$, the following conditions are equivalent:

(i) $\mathbf{S F}^{n}(M, N) \neq \emptyset$;

(ii) $N \in \Omega_{n}(M)$;

(iii) $\quad M \in \Omega_{-n}(N)$.

Moreover, when either $n \geq 2$ or $n=1$ and $G$ has the cancellation property for free modules, these conditions are equivalent to

(iv) $\operatorname{Free}^{n}(M, N) \neq \emptyset$.

Proof. We first assume that $n \geq 2$ and prove the equivalence of (i)-(iv). The equivalence of (ii) and (iii) is obvious, as are the implications (iv) $\Longrightarrow$ (i) and (iv) $\Longrightarrow$ (ii). Thus it suffices to show that (ii) $\Longrightarrow$ (i), and (i) $\Longrightarrow($ iv). 
(ii) $\Longrightarrow$ (i). First note that if $F$ is a finitely generated free module over $\mathbf{Z}[G]$, and $F_{1} \subset F$ is a free $\mathbf{Z}[G]$ submodule, then $F / F_{1}$ is stably free over $\mathbf{Z}[G]$ if and only if $F / F_{1}$ is torsion free over $\mathbf{Z}$; this is an easy argument using the injectivity of $F_{1}$ relative to $\mathcal{F}(\mathbf{Z}[G])$ or, what is the same, by double dualization using the universal property of free modules. Now suppose that $N \in \Omega_{n}(M)$; by definition, there exists a module $D$ which occurs in an exact sequence

$$
0 \rightarrow D \rightarrow \mathbf{Z}[G]^{\gamma} \rightarrow F_{n-2} \rightarrow \cdots \rightarrow F_{0} \rightarrow M \rightarrow 0 .
$$

with $\gamma>0$, each $F_{r}$ finitely generated free over $\mathbf{Z}[G]$, such that

$$
N \oplus \mathbf{Z}[G]^{a} \cong D \oplus \mathbf{Z}[G]^{b}
$$

for some $a, b \geq 0$. Now $D \oplus \mathbf{Z}[G]^{b}$ occurs in the exact sequence

$$
0 \rightarrow D \oplus \mathbf{Z}[G]^{b} \rightarrow \mathbf{Z}[G]^{\gamma+b} \rightarrow F_{n-2} \rightarrow \cdots \rightarrow F_{0} \rightarrow M \rightarrow 0,
$$

so that $N \oplus \mathbf{Z}[G]^{a}$ occurs in the exact sequence

$$
0 \rightarrow N \oplus \mathbf{Z}[G]^{a} \rightarrow \mathbf{Z}[G]^{\gamma+b} \rightarrow F_{n-2} \rightarrow \cdots \rightarrow F_{0} \rightarrow M \rightarrow 0,
$$

We split this last sequence as a pair thus:

$$
\begin{gathered}
0 \rightarrow N \oplus \mathbf{Z}[G]^{a} \rightarrow \mathbf{Z}[G]^{\gamma+b} \rightarrow K \rightarrow 0 \\
0 \rightarrow K \rightarrow F_{n-2} \rightarrow \cdots \rightarrow F_{0} \rightarrow M \rightarrow 0 .
\end{gathered}
$$

Dividing through (I) by $\mathbf{Z}[G]^{m}$ gives an extension

$$
0 \rightarrow N \rightarrow \mathbf{Z}[G]^{\gamma+b} / \mathbf{Z}[G]^{a} \rightarrow K \rightarrow 0
$$

in which $N, K \in \mathcal{F}(\mathbf{Z}[G])$. Thus, $\mathbf{Z}[G]^{\gamma+b} / \mathbf{Z}[G]^{a}$ is torsion free over $\mathbf{Z}$, so that, by the remark above, $\mathbf{Z}[G]^{\gamma+b} / \mathbf{Z}[G]^{a}=S$ is stably free over $\mathbf{Z}[G]$. Splicing (II) and (III) gives an element $0 \rightarrow N \rightarrow S \rightarrow F_{n-2} \rightarrow \cdots \cdots \rightarrow F_{0} \rightarrow M \rightarrow 0$, of $\mathbf{S F}^{n}(M, N)$ as required. This completes the proof that (ii) $\Longrightarrow$ (i).

(i) $\Longrightarrow$ (iv). Suppose that $0 \rightarrow N \rightarrow S_{n-1} \rightarrow \cdots \rightarrow S_{0} \rightarrow M \rightarrow 0$ is an element of $\mathbf{S F}^{n}(M, N)$ where each $S_{i}$ is finitely generated stably free. If $n=2$ (this is only place where we need the hypothesis that $n \geq 2$ ) then there exists $a>0$ so that $S_{i} \oplus \mathbf{Z}[G]^{a}$ is free for $i=1,2$. Adding a summand of $\mathbf{Z}[G]^{a}$ to the middle terms gives an extension

$$
0 \rightarrow N \rightarrow S_{1} \oplus \mathbf{Z}[G]^{a} \rightarrow S_{0} \oplus \mathbf{Z}[G]^{a} \rightarrow M \rightarrow 0
$$

which defines an element of $\mathbf{F r e e}^{2}(M, N)$. If $n>2$, we may first add a suitable free summand to $S_{1}$ and $S_{0}$ to make $S_{0}$ free; the result follows by induction after an easy splicing argument. This completes the proof that (i) $\Longrightarrow$ (iv).

Now suppose that $n=1$; again the equivalence of (ii) and (iii) is obvious, and the proof that (ii) $\Longrightarrow$ (i) given above goes through with a a minor change of indexing, so that it suffices to show that (i) $\Longrightarrow$ (ii). Thus suppose that $0 \rightarrow$ $N \rightarrow S \rightarrow M \rightarrow 0$ is an extension in which $S$ is a finitely generated stably free module. Then for some $a \geq 0, S \oplus \mathbf{Z}[G]^{a}$ is free, and from the exact sequence

$$
0 \rightarrow N \oplus \mathbf{Z}[G]^{a} \rightarrow S \oplus \mathbf{Z}[G]^{a} \rightarrow M \rightarrow 0
$$


we see that $N \oplus \mathbf{Z}[G]^{a} \in \Omega_{1}(M)$. Thus $N \in \Omega_{1}(M)$, showing that (i) $\Longrightarrow$ (ii), and hence the equivalence of (i), (ii) and (iii) in the case $n=1$.

Finally, if $G$ has the cancellation property for free modules then (i) is equivalent to (iv).

We denote by $\operatorname{Proj}^{n}(M, N)$ (resp. $\mathrm{SF}^{n}(M, N)$, $\operatorname{Free}^{n}(M, N)$ ) the image of $\operatorname{Proj}^{n}(M, N)\left(\right.$ resp. $\mathbf{S F}^{n}(M, N)$, Free $\left.{ }^{n}(M, N)\right)$ under the canonical map

$$
\operatorname{Ext}^{n}(M, N) \rightarrow \operatorname{Ext}^{n}(M, N) .
$$

Evidently $\operatorname{Free}^{n}(M, N) \subset \mathrm{SF}^{n}(M, N)$; one may extend the argument of (4.2) to show that for $n \geq 2, \mathbf{S F}^{n}(M, N)$ and $\mathbf{F r e e}^{n}(M, N)$ represent the same congruence classes; that is:

(4.3) For all $n \geq 2$, Free ${ }^{n}(M, N)=\operatorname{SF}^{n}(M, N)$.

For any finite group $G$, we proceed to give a description of $\operatorname{Ext}^{n}\left(\mathbf{Z}, \Omega_{n}(\mathbf{Z})\right) \cong$ $H^{n}\left(\mathbf{Z}, \Omega_{n}(\mathbf{Z})\right)$ when $\mathbf{Z}$ denotes the trivial module over $\mathbf{Z}[G]$ and $N \in \Omega_{n}(\mathbf{Z})$.

We begin by computing the ring $\operatorname{End}_{\mathcal{D} \text { er }}(\mathbf{Z})$. Note that $\operatorname{End}_{\mathbf{Z}[G]}(\mathbf{Z}) \cong \mathbf{Z}$ under the isomorphism $\alpha \mapsto \alpha(1)$; also there are $\mathbf{Z}[G]$-homomorphisms $\boldsymbol{\Sigma}: \mathbf{Z} \rightarrow \mathbf{Z}[G]$ and $\epsilon: \mathbf{Z}[G] \rightarrow \mathbf{Z}$ given by

$$
\boldsymbol{\Sigma}(n)=n\left(\sum_{g \in G} g\right) ; \epsilon\left(\sum_{g \in G} a_{g} g\right)=\sum_{g \in G} a_{g} .
$$

Moreover,

(4.4) If $\varphi: \mathbf{Z} \rightarrow \mathbf{Z}[G]$ is a $\mathbf{Z}[G]$-homomorphism then $\varphi=n \Sigma$ for some $n \in \mathbf{Z}$;

(4.5) If $\psi: \mathbf{Z}[G] \rightarrow \mathbf{Z}$ is a $\mathbf{Z}[G]$-homomorphism then $\varphi=n \epsilon$ for some $n \in \mathbf{Z}$.

Suppose $\alpha: \mathbf{Z} \rightarrow \mathbf{Z}$ factors through $\mathbf{Z}[G]^{m}$ for some $m \geq 1$ thus:

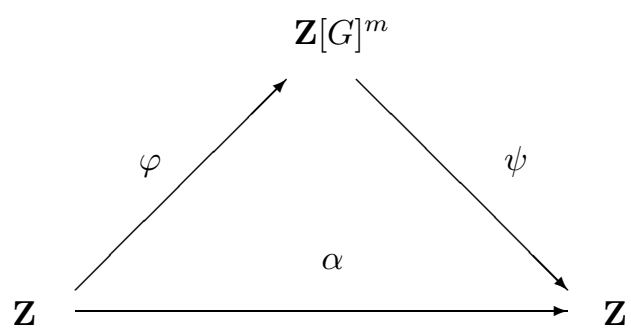


We may write $\varphi=\left(a_{1} \boldsymbol{\Sigma}, \cdots, a_{m} \boldsymbol{\Sigma}\right)$ and $\psi=\left(\begin{array}{c}b_{1} \epsilon \\ \vdots \\ b_{m} \epsilon\end{array}\right)$ so that

$$
\alpha=\left(\sum_{i} a_{i} b_{i}\right) \epsilon \boldsymbol{\Sigma}=\left(\sum_{i} a_{i} b_{i}\right)|G| .
$$

Conversely, if $\alpha=a|G|$ then $\alpha$ factors through $\mathbf{Z}[G]$ since $\alpha=a \epsilon \circ \boldsymbol{\Sigma}$. Thus we see that

(4.6) $\quad \alpha$ factors through some $\mathbf{Z}[G]^{m} \Longleftrightarrow \quad \alpha=n|G|$ for some $n$.

Clearly $\alpha$ factors through a projective if and only if it factors through some $\mathbf{Z}[G]^{n}$, so that

Proposition 4.7. Let $\mathbf{Z}$ denote the trivial module over $\mathbf{Z}[G]$ where $G$ is a finite group; then there is an isomorphism of rings

$$
\operatorname{End}_{\mathcal{D e r}}(\mathbf{Z}) \cong \mathbf{Z} /|G| \text {. }
$$

However, each $\Omega_{k}$ is a self-equivalence of the derived category ([13]), so that more generally we have:

Proposition 4.8. If $N \in \Omega_{k}(\mathbf{Z})$ then there is an isomorphism of rings

$$
\operatorname{End}_{\mathcal{D e r}}(N) \cong \mathbf{Z} /|G| \text {. }
$$

If $M \in \mathcal{F}(\mathbf{Z}[G])$, if $N \in \Omega_{n}(M)$ then $H^{n}(M, N) \cong H^{n}\left(M, \Omega_{n}(M)\right)$, and by the corepresentation formula $(3.3), H^{n}\left(M, \Omega_{n}(M)\right) \cong \operatorname{Hom}_{\mathcal{D} \operatorname{er}}\left(\Omega_{n}(M), \Omega_{n}(M)\right)$. Since $\Omega_{n}$ is a self-equivalence of $\operatorname{Der}(\mathcal{F}(\mathbf{Z}[G]))$,

$$
\operatorname{Hom}_{\mathcal{D} \text { er }}\left(\Omega_{n}(M), \Omega_{n}(M)\right) \cong \operatorname{Hom}_{\mathcal{D} \text { er }}(M, M) \cong \operatorname{End}_{\mathcal{D} \text { er }}(M) .
$$

Thus in the case where $M=\mathbf{Z}$ we obtain

$$
\operatorname{Ext}^{n}(\mathbf{Z}, N) \cong \mathbf{Z} /|G|
$$

when $N \in \Omega_{n}(\mathbf{Z})$, and more generally, by dimension shifting,

$$
\operatorname{Ext}^{n}(M, N) \cong \mathbf{Z} /|G|
$$

for $M \in \Omega_{m}(\mathbf{Z})$ and $N \in \Omega_{n}(M)$.

The explicit parametrization of the congruence classes in (4.10) is effected by the method of 'k-invariants' thus; let $M \in \Omega_{m}(\mathbf{Z})$ and $N \in \Omega_{n}(M)$. Since $N \in \Omega_{m+n}(\mathbf{Z})$, there is a ring isomorphism $\kappa: \operatorname{End}_{\mathcal{D e r}}(N) \rightarrow \mathbf{Z} /|G|$. If $\mathbf{P} \in \operatorname{Proj}^{n}(M, N)$ and $\mathbf{E}$ is an arbitrary element of $\mathbf{E x t}^{n}(M, N)$, the universal property of projective modules leads to a commutative diagram of $\mathbf{Z}[G]$-modules 
thus:

$$
\begin{aligned}
& \mathbf{P} \\
& \downarrow \alpha \\
& \mathbf{E}
\end{aligned}=\left(\begin{array}{cccc}
0 \rightarrow & N \rightarrow P_{n-1} \rightarrow \cdots \cdots \rightarrow P_{0} \rightarrow M \rightarrow 0 \\
& \downarrow \alpha_{+} \downarrow \alpha_{n-1} & \downarrow & \downarrow \alpha_{0} \downarrow \text { Id } \\
0 \rightarrow & N \rightarrow E_{n-1} \rightarrow \cdots \cdots \rightarrow E_{0} \rightarrow M \rightarrow 0
\end{array}\right) .
$$

We define the $k$-invariant of the transition, $k(\mathbf{P} \rightarrow \mathbf{E}) \in \mathbf{Z} /|G|$, by

$$
k(\mathbf{P} \rightarrow \mathbf{E})=\kappa\left(\alpha_{+}\right) .
$$

The k-invariant construction has the following easily verified properties:

Proposition 4.11. Let $\mathbf{P}, \mathbf{Q} \in \operatorname{Proj}^{n}(M, N)$, and let $\mathbf{E} \in \mathbf{E x t}^{n}(M, N)$; then

(i) $k(\mathbf{P} \rightarrow \mathbf{E})=k(\mathbf{P} \rightarrow \mathbf{Q}) k(\mathbf{Q} \rightarrow \mathbf{E})$

(ii) $k(\mathbf{P} \rightarrow \mathbf{P})=1$;

(iii) $k(\mathbf{P} \rightarrow \mathbf{Q}) k(\mathbf{Q} \rightarrow \mathbf{P})=1$;

(iv) $\mathbf{E} \in \mathbf{P r o j}^{n}(M, N) \Longleftrightarrow k(\mathbf{P} \rightarrow \mathbf{E}) \in(\mathbf{Z} /|G|)^{*}$.

Making a specific choice of projective extension $\mathbf{P} \in \mathbf{P r o j}^{n}(M, N)$ we see that:

Proposition 4.12. The correspondence $\mathbf{E} \mapsto k(\mathbf{P} \rightarrow \mathbf{E})$ defines a bijective mapping $\operatorname{Proj}^{n}(M, N) \rightarrow(\mathbf{Z} /|G|)^{*}$ for $M \in \Omega_{m}(\mathbf{Z})$ and $N \in \Omega_{n}(M)$.

\section{Algebraic 2-complexes}

If $K$ is a finite connected 2-complex with $\pi_{1}(K)=G$, then the universal covering $\tilde{K}$ is simply connected, so that the cellular chain complex $C_{*}(\tilde{K})$ can be written as an exact sequence of $\mathbf{Z}[G]$ modules thus:

$$
C_{*}(\tilde{K})=\left(0 \rightarrow \pi_{2}(K) \rightarrow C_{2}(\tilde{K}) \stackrel{\partial_{2}}{\rightarrow} C_{1}(\tilde{K}) \stackrel{\partial_{1}}{\rightarrow} C_{0}(\tilde{K}) \rightarrow \mathbf{Z} \rightarrow 0\right) .
$$

In particular, the second homotopy group $\pi_{2}(K)$ is naturally a $\mathbf{Z}[G]$-module belonging to the stable module $\Omega_{3}(\mathbf{Z})$, and the cell complex itself determines an element of $\mathbf{F r e e}^{3}\left(\mathbf{Z}, \pi_{2}(K)\right)$. We have seen already in (4.3) that the set $\operatorname{Free}^{3}\left(\mathbf{Z}, \pi_{2}(K)\right)$, of congruence classes of free extensions coincides with the corresponding set $\mathrm{SF}^{3}\left(\mathbf{Z}, \pi_{2}(K)\right)$ formed from stably free extensions. By an algebraic 2-complex we shall mean an extension of the form $\mathbf{E}=\left(0 \rightarrow J \stackrel{j}{\rightarrow} E_{2} \stackrel{\partial_{2}}{\rightarrow} E_{1} \stackrel{\partial_{1}}{\rightarrow} E_{0} \rightarrow \mathbf{Z} \rightarrow 0\right)$ where each $E_{i}$ is finitely generated stably free; in particular, $J \in \Omega_{3}(\mathbf{Z})$ and $\mathbf{E} \in \mathbf{S F}^{3}(\mathbf{Z}, J)$.

For any $n \geq 1$, the $n$-fold stabilization of $\mathbf{E}$ is the complex

$$
\mathcal{S}^{n}(\mathbf{E})=\left(0 \rightarrow J \oplus \mathbf{Z}[G]^{n} \stackrel{j \oplus \mathrm{Id}}{\longrightarrow} E_{2} \oplus \mathbf{Z}[G]^{n} \stackrel{\partial_{2} \circ \pi}{\longrightarrow} E_{1} \stackrel{\partial_{1}}{\rightarrow} E_{0} \rightarrow \mathbf{Z} \rightarrow 0\right)
$$

where $\pi: E_{2} \oplus \mathbf{Z}[G]^{n} \rightarrow E_{2}$ is the projection.

This corresponds to the following geometrical construction; if $K$ is a finite 2complex, we denote by $\Sigma^{n}(K)$ the complex obtained from the one point union of 
$K$ with a collection of $n$ copies of the 2-sphere;

$$
\Sigma^{n}(K)=K \vee \underbrace{S^{2} \vee \cdots \vee S^{2}}_{n} .
$$

It is straightforward to check that $C_{*}\left(\widetilde{\Sigma^{n}(K)}\right) \cong \mathcal{S}^{n}\left(C_{*}(\tilde{K})\right)$.

The discussion can be carried out in the context of group presentations: if $\mathcal{G}=\left\langle x_{1}, \cdots, x_{g} \mid W_{1}, \cdots, W_{r}\right\rangle$ is a finite presentation of $G$, we denote by $C_{*}(\mathcal{G})$ the cellular chain complex of $\widetilde{\mathcal{K}_{\mathcal{G}}}$ where $\mathcal{K}_{\mathcal{G}}$ is the Cayley complex of $\mathcal{G}$ :

$$
C_{*}(\mathcal{G})=\left(0 \rightarrow \pi_{2}(\mathcal{G}) \rightarrow C_{2}\left(\widetilde{\mathcal{K}_{\mathcal{G}}}\right) \stackrel{\partial_{2}}{\rightarrow} C_{1}\left(\widetilde{\mathcal{K}_{\mathcal{G}}}\right) \stackrel{\partial_{1}}{\rightarrow} \mathbf{Z}[G] \stackrel{\epsilon}{\rightarrow} \mathbf{Z} \rightarrow 0\right)
$$

It is clear from Schanuel's Lemma that the module $\pi_{2}(\mathcal{G})=\pi_{2}\left(K_{\mathcal{G}}\right)$ is stably unique and belongs to $\Omega_{3}(\mathbf{Z})$. If we wish to emphasise the group presentational aspects, as against the module theoretic aspects, of the situation we could also, as was essentially done by Fox [9], observe that the stable uniqueness of $\pi_{2}(\mathcal{G})$ already follows from Tietze's Theorem [28]. In any case, denoting by $\pi_{2}(G)$ the stable module $\left[\pi_{2}(\mathcal{G})\right]$, we have:

Proposition 5.1. For any finite group $G, \pi_{2}(G)=\Omega_{3}(\mathbf{Z})$.

The process of stabilization also has an interpretation in terms of group presentations; we denote by $\Sigma^{n} \mathcal{G}$ any presentation of $G$ obtained from $\mathcal{G}$ by adding $n$ trivial relations. It is straightforward to see that $C_{*}\left(\Sigma^{n} \mathcal{G}\right)$ is congruent to $\mathcal{S}^{n}\left(C_{*}(\mathcal{G})\right)$.

A morphism $h: \mathbf{E} \rightarrow \mathbf{F}$ in $\mathbf{E x t}^{3}(M, N)$ is said to be a homotopy equivalence (strictly speaking, a weak homotopy equivalence) when $h_{M}=I d$ and $h_{N}$ is an isomorphism; that is, when it has the form

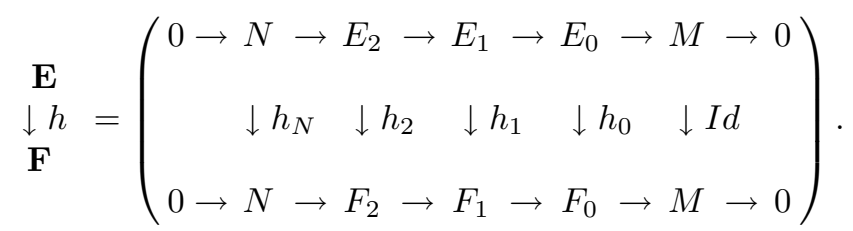

We write $\mathbf{E} \simeq \mathbf{F}$ when there exists a weak homotopy equivalence $h: \mathbf{E} \rightarrow \mathbf{F}$. Weak homotopy equivalence is always reflexive and transitive, but is not, in general, symmetric. If $h: \mathbf{E} \rightarrow \mathbf{F}$ is a weak homotopy equivalence, the mapping cone $M(h)$ is necessarily acyclic; if, in addition, $\mathbf{E}, \mathbf{F}$ are projective then $M(h)$ is chain contractible. By the usual argument ([21] p. 167), $h$ then has an inverse up to homotopy. Thus 'weak homotopy equivalence' is indeed an equivalence relation when restricted to $\mathbf{P r o j}^{n}(\mathbf{Z}, J)$.

A module $J \in \Omega_{3}(\mathbf{Z})$ is said to be minimal when $\operatorname{rk}_{\mathbf{Z}}(J) \leq \operatorname{rk}_{\mathbf{Z}}\left(J^{\prime}\right)$ for all $J^{\prime} \in \Omega_{3}(\mathbf{Z})$. If $J \in \Omega_{3}(\mathbf{Z})$ and $\mathbf{E}=\left(0 \rightarrow J \rightarrow F_{2} \rightarrow F_{1} \rightarrow F_{0} \rightarrow \mathbf{Z} \rightarrow 0\right)$ is an algebraic 2-complex then $\mathbf{E}$ is said to be minimal when $J$ is minimal. $G$ is said to be 2-tame (resp. minimally 2-tame) when for every algebraic 2-complex 
$\mathbf{E}$ (resp. every minimal algebraic 2-complex $\mathbf{E}$ ) there exists a presentation $\mathcal{G}$ such that $C_{*}(\mathcal{G}) \simeq \mathbf{E}$.

Proposition 5.2. Let $G$ be a finite group; then

$$
G \text { is } 2 \text {-tame } \Longleftrightarrow G \text { is minimally } 2 \text {-tame. }
$$

Proof. The implication $(\Longrightarrow)$ is clear. Thus suppose that $G$ is minimally 2-tame, let $J \in \Omega_{3}(\mathbf{Z})$ be a specific choice of minimal module, and let

$$
\mathbf{F}=\left(0 \rightarrow J \stackrel{j}{\rightarrow} F_{2} \stackrel{\partial_{2}}{\rightarrow} F_{1} \stackrel{\partial_{1}}{\rightarrow} F_{0} \rightarrow \mathbf{Z} \rightarrow 0\right)
$$

be a minimal algebraic 2-complex whose left-hand end is $J$ but whose choice is otherwise arbitrary. Now let

$$
\mathbf{E}=\left(0 \rightarrow \Lambda \rightarrow E_{2} \rightarrow E_{1} \rightarrow E_{0} \rightarrow \mathbf{Z} \rightarrow 0\right)
$$

be a non-minimal free algebraic 2-complex. By the Swan-Jacobinski Theorem, we may suppose, without any loss of generality, that $\Lambda=J \oplus \mathbf{Z}[G]^{n}$. Put

$$
r=k\left(\mathcal{S}^{n}(\mathbf{F}) \rightarrow \mathbf{E}\right) \in(\mathbf{Z} /|G|)^{*} .
$$

Let $\mathbf{D}=\left(0 \rightarrow J \rightarrow D_{2} \rightarrow D_{1} \rightarrow D_{0} \rightarrow \mathbf{Z} \rightarrow 0\right)$ be an element of $\mathbf{E x t}^{3}(\mathbf{Z}, J)$ such that

$$
k(\mathbf{F} \rightarrow \mathbf{D})=r .
$$

Since $r \in(\mathbf{Z} /|G|)^{*}$ it follows that $\mathbf{D} \in \mathbf{P r o j}^{3}(\mathbf{Z}, J)$. Furthermore, we may make the identifiction

$$
\operatorname{Ext}^{3}\left(\mathbf{Z}, J \oplus \mathbf{Z}[G]^{n}\right) \cong \operatorname{Ext}^{3}\left(\mathbf{Z}, J \oplus \mathbf{Z}[G]^{n}\right) \cong(\mathbf{Z} /|G|)^{*} .
$$

Since $k\left(\mathcal{S}^{n}(\mathbf{F}) \rightarrow \mathbf{E}\right)=k(\mathbf{F} \rightarrow \mathbf{D})=r$, and $\mathbf{E}$ is free, it follows that $\mathbf{D}$ is stably free, and hence a minimal algebraic 2-complex. By hypothesis, there exists a presentation $\mathcal{G}$ such that $C_{*}(\mathcal{G}) \simeq \mathbf{D}$. However $k\left(\mathcal{S}^{n} \mathbf{F} \rightarrow \mathcal{S}^{n} \mathbf{D}\right)=r$, so that $\mathcal{S}^{n} \mathbf{D}$ is congruent to $\mathbf{E}$ and hence $\mathcal{S}^{n} \mathbf{D} \simeq \mathbf{E}$. Let $\Sigma^{n} \mathcal{G}$ be a presentation obtained from $\mathcal{G}$ by adding $n$ trivial relations. Then $C_{*}\left(\Sigma^{n} \mathcal{G}\right) \simeq \mathcal{S}^{n}(\mathbf{D}) \simeq \mathbf{E}$.

For algebraic 2-complexes, and more generally for projective complexes, the relation 'weak homotopy equivalence' is symmetrical; that is, if $\mathbf{E}, \mathbf{E}^{\prime}$ are algebraic 2-complexes, and $f: \mathbf{E} \rightarrow \mathbf{E}^{\prime}$ is a homotopy equivalence, then there exists an inverse homotopy equivalence $g: \mathbf{E}^{\prime} \rightarrow \mathbf{E}$. If $\mathbf{E}$ is an algebraic 2-complex over $\mathbf{Z}[G]$, say that $\mathbf{E}$ is geometrically realized when there is a finite 2-complex $L$ with $\pi_{1}(L)=G$, and a homotopy equivalence $\varphi: C_{*}(L) \stackrel{\sim}{\rightarrow} \mathbf{E}$; equivalently, when there is a finite presentation $\mathcal{G}$ of $G$ and a homotopy equivalence $\varphi: C_{*}(\mathcal{G}) \stackrel{\sim}{\rightarrow} \mathbf{E}$. A finite group $G$ is said to be 2-trivial when there exists a single presentation $\mathcal{G}$ of $G$ such that $\mathbf{E} \simeq C_{*}(\mathcal{G})$ for each minimal algebraic 2-complex $\mathbf{E}$. It is straightforward to see that

Proposition 5.3. If $G$ is 2-trivial then $G$ is 2-tame. 
At present, it is still unknown whether every finite group $G$ is necessarily 2tame. However, we point out that the condition of 2-triviality imposes strong requirements on $G$. In particular, the stable module $\Omega_{3}(\mathbf{Z})$ must then contain a unique module at the minimal level, so that the tree structure has no branching at all. Browning [4] classified the minimal 2-complexes of finite abelian groups, and showed that they arise from suitable presentations; thus finite abelian groups are 2-tame, but, by results of Metzler [20], definitely not 2-trivial.

\section{Virtual homotopy type and the $\mathrm{D}(2)$-problem}

We now proceed to reduce the $\mathrm{D}(2)$-problem, for finite fundamental groups, to a question involving only 2-dimensional algebraic homotopy types.

Proposition 6.1. Let $X$ be a finite 3 -complex with $\pi_{1}(X) \cong G$, and put $K=$ $X^{(2)}$; then there is a canonical exact sequence of $\mathbf{Z}[G]$-modules

$$
0 \rightarrow H_{3}(\tilde{X} ; \mathbf{Z}) \rightarrow C_{3}(\tilde{X}) \stackrel{\partial_{3}}{\rightarrow} \pi_{2}(K) \rightarrow \pi_{2}(X) \rightarrow 0
$$

Proof. We have a pair of exact sequences

$$
\begin{gathered}
0 \rightarrow Z_{3}(\tilde{X}) \rightarrow C_{3}(\tilde{X}) \stackrel{\partial_{3}}{\rightarrow} \operatorname{Im}\left(\partial_{3}\right) \rightarrow 0 \\
0 \rightarrow \operatorname{Im}\left(\partial_{3}\right) \rightarrow Z_{2}(\tilde{X}) \rightarrow H_{2}(\tilde{X} ; \mathbf{Z}) \rightarrow 0
\end{gathered}
$$

which, spliced together give:

$$
0 \rightarrow Z_{3}(\tilde{X}) \rightarrow C_{3}(\tilde{X}) \stackrel{\partial_{3}}{\rightarrow} Z_{2}(\tilde{X}) \rightarrow H_{2}(\tilde{X} ; \mathbf{Z}) \rightarrow 0 .
$$

Since $K=X^{(2)}$ we have $H_{2}(\tilde{K} ; \mathbf{Z})=Z_{2}(\tilde{K})=Z_{2}(\tilde{X})$, whilst $Z_{3}(\tilde{X})=H_{3}(\tilde{X} ; \mathbf{Z})$, so that we have an exact sequence

$$
0 \rightarrow H_{3}(\tilde{X} ; \mathbf{Z}) \rightarrow C_{3}(\tilde{X}) \stackrel{\partial_{3}}{\rightarrow} H_{2}(\tilde{K} ; \mathbf{Z}) \rightarrow H_{2}(\tilde{X} ; \mathbf{Z}) \rightarrow 0 .
$$

The result now follows from the Hurewicz Theorem, since $\pi_{2}(K) \cong \pi_{2}(\tilde{K})=$ $H_{2}(\tilde{K} ; \mathbf{Z})$ and $\pi_{2}(X) \cong \pi_{2}(\tilde{X})=H_{2}(\tilde{X} ; \mathbf{Z})$.

As a consequence, we have:

Proposition 6.2. Let $X$ be a finite 3 -complex with $\pi_{1}(X) \cong G$; if $H^{3}(X ; \mathcal{B})=0$ for any local coefficient system $\mathcal{B}$ on $X$ then

(i) $\operatorname{Im}\left(\partial_{3}\right)$ is isomorphic to the free $\mathbf{Z}[G]$-module $C_{3}(\tilde{X})$;

(ii) $\operatorname{Im}\left(\partial_{3}\right)$ is a direct summand of $\pi_{2}(K)$, and

(iii) $\pi_{2}(X) \cong \pi_{2}(K) / \operatorname{Im}\left(\partial_{3}\right)$. 
Proof. Since $X$ is three dimensional, $H_{3}(\tilde{X} ; \mathbf{Z})$ is torsion free, since by the Universal Coefficient Theorem, $\operatorname{Tor}\left(H_{3}(\tilde{X} ; \mathbf{Z})\right)=\operatorname{Tor}\left(H^{4}(\tilde{X} ; \mathbf{Z})\right)=0$. Moreover, by the cohomology hypothesis on $X$, we have $H_{3}(\tilde{X} ; \mathbf{Q})=H^{3}(\tilde{X} ; \mathbf{Q})=0$. It follows that $H_{3}(\tilde{X} ; \mathbf{Z})=0$. Thus $\partial_{3}: C_{3}(\tilde{X}) \rightarrow \operatorname{Im}\left(\partial_{3}\right)$ is an isomorphism. This proves (i).

Furthermore, the exact sequence of (6.1) reduces to

$$
0 \rightarrow C_{3}(\tilde{X}) \stackrel{\partial_{3}}{\rightarrow} \pi_{2}(K) \rightarrow \pi_{2}(X) \rightarrow 0
$$

However,

$$
\operatorname{Tor}\left(\pi_{2}(X)\right)=\operatorname{Tor}\left(H_{2}(\tilde{X} ; \mathbf{Z})\right)=\operatorname{Tor}\left(H^{3}(\tilde{X} ; \mathbf{Z})\right)
$$

so that, again by the cohomology assumption on $X, \pi_{2}(X)$ is torsion free. Thus we have a short exact sequence in $\mathcal{F}(\mathbf{Z}[G])$

$$
0 \rightarrow C_{3}(\tilde{X}) \stackrel{\partial_{3}}{\rightarrow} \pi_{2}(K) \rightarrow \pi_{2}(X) \rightarrow 0
$$

in which $C_{3}(\tilde{X}) \cong \operatorname{Im}\left(\partial_{3}\right)$ is $\mathbf{Z}[G]$-free (of rank $n$ say) and hence relatively injective. Thus

$$
\pi_{2}(K) \cong \pi_{2}(X) \oplus \operatorname{Im}\left(\partial_{3}\right) \cong \pi_{2}(X) \oplus \mathbf{Z}[G]^{n} .
$$

This proves (ii) and (iii) simultaneously.

It follows from the above that:

Corollary 6.3. Let $X$ be a finite 3 -complex with $\pi_{1}(X) \cong G$; if $H^{3}(X ; \mathcal{B})=0$ for any local coefficient system $\mathcal{B}$ on $X$ then $\pi_{2}(X) \in \Omega_{3}(\mathbf{Z})$.

Throughout the rest of this section, until further notice, $X$ will denote a finite 3-complex with $\pi_{1}(X) \cong G$ which is cohomologically 2-dimensional in the sense that $\left.H^{3}(X ; \mathcal{B})\right)=0$ for any local coefficient system $\mathcal{B}$ on $X$. Up to homotopy type, there is no loss of generality in assuming that $X$ is also reduced, so we assume that as well. $K$ will denote the 2 -skeleton of $X$. We may refine the above analysis slightly. Firstly, since $H_{3}(\tilde{X} ; \mathbf{Z})=0$ the boundary map $\partial_{3}: C_{3}(\tilde{X}) \rightarrow C_{2}(\tilde{X})$ is injective. Moreover, we have:

Proposition 6.4. $\pi_{2}(X) \cong \operatorname{Ker}\left(\partial_{2}: C_{2}(\tilde{X}) / \operatorname{Im}\left(\partial_{3}\right) \rightarrow C_{1}(\tilde{X})\right.$.

Proof. This follows directly from the exactness of the following sequence of $\mathbf{Z}[G]$ modules $0 \rightarrow \pi_{2}(K) / \operatorname{Im}\left(\partial_{3}\right) \rightarrow C_{2}(\tilde{X}) / \operatorname{Im}\left(\partial_{3}\right) \stackrel{\partial_{2}}{\rightarrow} C_{1}(\tilde{X})$.

Proposition 6.5. $\operatorname{Im}\left(\partial_{3}\right)$ is a direct summand of $C_{2}(\tilde{X})$ and $C_{2}(\tilde{X}) / \operatorname{Im}\left(\partial_{3}\right)$ is stably free over $\mathbf{Z}[G]$.

Proof. We have an exact sequence $0 \rightarrow \pi_{2}(X) \rightarrow C_{2}(\tilde{X}) / \operatorname{Im}\left(\partial_{3}\right) \stackrel{\partial_{2}}{\rightarrow} C_{1}(\tilde{X})$ in which $\pi_{2}(X)$, being a representative of $\Omega_{3}(\mathbf{Z})$, is torsion free over $\mathbf{Z}$. However, 
$C_{1}(\tilde{X})$ is free over $\mathbf{Z}[G]$ and hence free over $\mathbf{Z}$. Thus $C_{2}(\tilde{X}) / \operatorname{Im}\left(\partial_{3}\right)$ is also torsion free over $\mathbf{Z}$. The result now follows from the injectivity of the free module $\operatorname{Im}\left(\partial_{3}\right)$ relative to $\mathcal{F}(\mathbf{Z}[G])$, given that $C_{2}(\tilde{X})$ is also free.

Let $j: \pi_{2}(X) \rightarrow C_{2}(\tilde{X}) / \operatorname{Im}\left(\partial_{3}\right)$ be the inclusion obtained by making the identifications

$$
\pi_{2}(X)=\pi_{2}(K) / \operatorname{Im}\left(\partial_{3}\right) ; \quad C_{2}(\tilde{X})=C_{2}(\tilde{K}) .
$$

We let $\langle X\rangle$ denote the following algebraic 2-complex;

$$
0 \rightarrow \pi_{2}(X) \stackrel{j}{\rightarrow} C_{2}(\tilde{X}) / \operatorname{Im}\left(\partial_{3}\right) \rightarrow C_{1}(\tilde{X}) \rightarrow C_{0}(\tilde{X}) \rightarrow \mathbf{Z} \rightarrow 0 .
$$

Observe that $\langle X\rangle$ is functorial in the cell structure of $X$. Clearly $\langle X\rangle$ belongs to $\mathbf{S F}^{3}\left(\mathbf{Z}, \pi_{2}(X)\right)$, and we regard it as a 'virtual' 2-dimensional homotopy type representing $X$. There is a natural $\mathbf{Z}[G]$-chain map $\varphi: C_{*}(X) \rightarrow\langle X\rangle$ from the cellular chain complex of $X$ obtained by collapsing $C_{3}(\tilde{X}) \cong \operatorname{Im}\left(\partial_{3}\right)$ :

$$
\begin{aligned}
& 0 \rightarrow C_{3}(\tilde{X}) \rightarrow \quad C_{2}(\tilde{X}) \quad \rightarrow C_{1}(\tilde{X}) \rightarrow C_{0}(\tilde{X}) \rightarrow \mathbf{Z} \rightarrow 0 \\
& 0 \rightarrow C_{2}(\tilde{X}) / \operatorname{Im}\left(\partial_{3}\right) \rightarrow C_{1}(\tilde{X}) \rightarrow C_{0}(\tilde{X}) \rightarrow \mathbf{Z} \rightarrow 0
\end{aligned}
$$

where, since $X$ is reduced, we may identify the epimorphism $C_{0}(\tilde{X}) \rightarrow \mathbf{Z}$ with the augmentation map.

By the Hurewicz Theorem, we may identify $\pi_{2}(X)$ with $H_{2}(\tilde{X} ; \mathbf{Z})$. The 'algebraic second homotopy group' of $\langle X\rangle$ is given by

$$
\pi_{2}(\langle X\rangle)=\pi_{2}(K) / \operatorname{Im}\left(\partial_{3}\right) .
$$

It is straightforward to check that

Proposition 6.6. $\varphi: C_{*}(X) \rightarrow\langle X\rangle$ induces an isomorphism

$$
\varphi: \pi_{2}(X) \stackrel{\simeq}{\rightarrow} \pi_{2}(\langle X\rangle) .
$$

We are tempted to restate (6.6) as saying that $\varphi: C_{*}(X) \rightarrow\langle X\rangle$ is a weak homotopy equivalence. The difficulty is that $C_{*}(X)$ and $\langle X\rangle$ are slightly different sorts of objects; $\langle X\rangle$ is both augmented (by $C_{0}(\tilde{X}) \rightarrow \mathbf{Z}$ ) and co-augmented (by $\left.\pi_{2}(X) \rightarrow C_{2}(\tilde{X})\right)$ whereas $C_{*}(X)$ is merely augmented. In comparing the two, we should, strictly speaking, drop the co-augmentation (which will then be remembered simply as $\operatorname{Ker}\left(\partial_{2}: C_{2}(\tilde{X}) / \operatorname{Im}\left(\partial_{3}\right) \rightarrow C_{1}(\tilde{X})\right)$. There is a transformation of (augmented, coaugmented) chain complexes $\nu: C_{*}(K) \rightarrow\langle X\rangle$, natural in the cell structure of $X$, described by the following diagram:

$$
\begin{array}{rlrlrl}
0 \rightarrow \quad \pi_{2}(K) & \rightarrow \quad C_{2}(\tilde{K}) & \rightarrow C_{1}(\tilde{K}) \rightarrow C_{0}(\tilde{K}) \rightarrow \mathbf{Z} \rightarrow 0 \\
& \downarrow \nu & \downarrow \nu_{2} & \downarrow I d & \downarrow I d & \downarrow I d \\
0 \rightarrow \pi_{2}(K) / \operatorname{Im}\left(\partial_{3}\right) \rightarrow C_{2}(\tilde{X}) / \operatorname{Im}\left(\partial_{3}\right) & \rightarrow C_{1}(\tilde{X}) \rightarrow C_{0}(\tilde{X}) \rightarrow \mathbf{Z} \rightarrow 0 .
\end{array}
$$


Theorem 6.7. Let $G$ be a finite group, and let $X$ be a finite cohomologically 2dimensional 3-complex with $\pi_{1}(X) \cong G$; then $X$ is homotopy equivalent to a finite 2-complex if and only if the algebraic 2-complex $\langle X\rangle$ is geometrically realized.

Proof. Suppose that $L$ is a finite 2-complex, and that $\psi: L \rightarrow X$ is a homotopy equivalence over the identity. Then $\psi: \pi_{2}(L) \rightarrow \pi_{2}(X)$ is an isomorphism. Hence $\varphi \circ \psi_{*}: \pi_{2}(L) \stackrel{\simeq}{\rightarrow} \pi_{2}(\langle X\rangle)$ is an isomorphism; thus $\varphi \circ \psi_{*}: C_{*}(L) \rightarrow\langle X\rangle$ is a weak homotopy equivalence, and so $\langle X\rangle$ is geometrically realized.

Conversely, suppose that $\langle X\rangle$ is geometrically realized. This means there is a finite 2-complex $L$ with $\pi_{1}(L)=G$ and a weak homotopy equivalence $g: C_{*}(L) \rightarrow$ $\langle X\rangle$. By symmetry of weak homotopy equivalence on algebraic 2-complexes, we may suppose there is also a weak homotopy equivalence $f:\langle X\rangle \rightarrow C_{*}(L)$. As usual, let $K$ denote the 2-skeleton of $X$; without loss of generality, we may suppose that:

(i) $L^{(1)}=K^{(1)}$,

and that

(ii) $f_{r}=\operatorname{Id}: C_{r}(\tilde{K}) \equiv C_{r}(\tilde{\mathcal{L}})$ for $r \leq 1$.

Furthermore, assuming $X$ has $N$ cells of dimension three, we may write:

$$
X=K \cup_{\alpha_{1}} E_{1}^{(3)} \cup_{\alpha_{2}} E_{2}^{(3)} \cdots \cdots \cup_{\alpha_{N}} E_{N}^{(3)}
$$

Observe that $f$ induces an isomorphism $f_{*}: \pi_{2}(\langle X\rangle)=\pi_{2}(X) \stackrel{\simeq}{\rightarrow} \pi_{2}(L)$. The chain map $f \circ \nu: C_{*}(K) \rightarrow C_{*}(L)$ has the property that $(f \circ \nu)_{r}=\operatorname{Id}: C_{r}(\tilde{K}) \equiv$ $C_{r}(\tilde{L})$ for $r \leq 1$. By $(2.3)$ there exists a cellular map $g: K \rightarrow L$ such that

(i) $f_{*}=I d: \pi_{1}(K) \rightarrow \pi_{1}(L)$, and

(ii) $g_{*}=f_{*} \circ \nu_{*}: \pi_{2}(K) \rightarrow \pi_{2}(L)$.

In particular, since $f_{*}$ is an isomorphism, we obtain.

$\operatorname{Ker}\left(g_{*}: \pi_{2}(K) \rightarrow \pi_{2}(L)\right)=\operatorname{Ker}\left(\nu_{*}\right)=\operatorname{Im}\left(\partial_{3}: C_{3}(\tilde{X}) \rightarrow \pi_{2}(K)\right) \cong \mathbf{Z}[G]^{N}$.

Each homotopy class $\left[\alpha_{j}\right]$ belongs to $\operatorname{Im}\left(\partial_{3}\right)=\operatorname{Ker}\left(g i_{*}: \pi_{2}(K) \rightarrow \pi_{2}(L)\right)$, so that $g: K \rightarrow L$ extends over the 3 -cells of $X$ to a map $h: X \rightarrow L$ such that

(iii) $h_{*}=I d: \pi_{1}(X) \rightarrow \pi_{1}(L)$ and

(iv) $h_{*}=f_{*}: \pi_{2}(X) \rightarrow \pi_{2}(L)$.

Since $H_{r}(\tilde{X} ; \mathbf{Z})=H_{r}(\tilde{L} ; \mathbf{Z})=0$ for $2<r$, we see by Whitehead's Theorem that $h$ is a homotopy equivalence as required.

A 2-tame group $G$ is precisely one over which every algebraic 2-complex is geometrically realized; thus we obtain:

Corollary 6.8. If $G$ is 2-tame then the D(2)-property holds for $G$.

In [16], we give examples of finite groups which are 2-tame; in the absence of positive evidence to the contrary, one can presently hope that every finite group 
is 2-tame, and so possesses the $\mathrm{D}(2)$-property. Nevertheless, the possibility of a constructing a counterexample is an intriguing one. So far, we have had no recourse to Browing's results. However, using the first of Browning's results, the Stability Theorem, we can establish the converse to (6.7). First we need a criterion for recognising cohomologically 2-dimensional 3-complexes. We adopt the following notation:

$K$ : a finite 2-complex with $\pi_{1}(K) \cong G$;

$m$ : an integer $\geq 1$;

$\alpha$ : a $\mathbf{Z}[G]$-homomorphism $\mathbf{Z}[G]^{m} \rightarrow \pi_{2}(K)$ will be;

$\left\{\epsilon_{j}\right\}_{1 \leq j \leq m}$ : the canonical $\mathbf{Z}[G]$-basis for $\mathbf{Z}[G]^{m}$;

$\alpha_{j}$ : a map $S^{2} \rightarrow K$ in the homotopy class $\alpha\left(\epsilon_{j}\right)$.

Furthermore, $X(\alpha)$ will denote the 3 -complex obtained by attaching 3-cells to $K$ by means of $\alpha_{1}, \alpha_{2}, \cdots, \alpha_{m}$; that is,

$$
X(\alpha)=K \cup_{\alpha_{1}} E_{1}^{(3)} \cup_{\alpha_{2}} E_{2}^{(3)} \cdots \cdots \cup_{\alpha_{m}} E_{m}^{(3)} .
$$

Lemma 6.9. Let $K$ be a finite 2 -complex with $\pi_{1}(K) \cong G$, and let $\alpha: \mathbf{Z}[G]^{m} \rightarrow$ $\pi_{2}(\mathcal{K})$ be an injective $\mathbf{Z}[G]$-homomorphism such that $\pi_{2}(\mathcal{K}) / \operatorname{Im}(\alpha)$ is torsion free over $\mathbf{Z}$. Then $H^{3}(X(\alpha) ; \mathcal{B})=0$ for all local coefficient systems $\mathcal{B}$ on $X(\alpha)$.

Proof. We can make the following identifications:

$$
\begin{array}{ccc}
C_{3}(\widetilde{X(\alpha)}) & \longleftrightarrow & \mathbf{Z}[G]^{m} \\
C_{2}(\widetilde{X(\alpha)}) & \longleftrightarrow & C_{2}(\tilde{\mathcal{K}}) \\
\pi_{2}(K) & \longleftrightarrow & Z_{2}(\tilde{K}) \\
\partial_{3}: C_{3}(\widetilde{X(\alpha)}) \rightarrow C_{2}(\widetilde{X(\alpha)}) & \longleftrightarrow & i \circ \alpha: \mathbf{Z}[G]^{m} \rightarrow C_{2}(\tilde{K}) \\
H^{3}(X(\alpha) ; \mathcal{B}) & \longleftrightarrow\left(\mathbf{Z}[G]^{M}\right)^{*} \otimes \mathcal{B} / \operatorname{Im}\left((i \circ \alpha)^{*} \otimes 1_{\mathcal{B}}\right) .
\end{array}
$$

where $i: \pi_{2}(K)=Z_{2}(\tilde{K}) \subset C_{2}(\tilde{K})$ is the inclusion. Since $\alpha$ is injective and $\pi_{2}(K) / \operatorname{Im}(\alpha)$ is $\mathbf{Z}$-free, the exact sequence

$$
0 \rightarrow \mathbf{Z}[G]^{m} \stackrel{\alpha}{\rightarrow} \pi_{2}(K) \rightarrow \pi_{2}(K) / \operatorname{Im}(\alpha) \rightarrow 0
$$

lies within $\mathcal{F}(\mathbf{Z})[G]$; hence its dual sequence is exact, and $\alpha^{*}: \pi_{2}(K)^{*} \rightarrow\left(\mathbf{Z}[G]^{m}\right)^{*}$ is surjective. Likewise, the exact sequence

$$
0 \rightarrow \pi_{2}(K) \rightarrow C_{2}(\tilde{K}) \rightarrow C_{1}(\tilde{K}) \rightarrow C_{0}(\tilde{K}) \rightarrow \mathbf{Z} \rightarrow 0
$$

lies within $\mathcal{F}(\mathbf{Z})[G]$, so its dual sequence too is exact, and $\imath^{*}: C_{2}(\tilde{K})^{*} \rightarrow \pi_{2}(K)^{*}$ is surjective. Hence $(\alpha \circ i)^{*}: C_{2}(\tilde{\mathcal{K}})^{*} \rightarrow\left(\mathbf{Z}[G]^{m}\right)^{*}$ is surjective. Thus $(i \circ \alpha)^{*} \otimes 1_{\mathcal{B}}$ : 
$\left.C_{2}(\tilde{K})^{*} \otimes \mathcal{B} \longrightarrow \mathbf{Z}[G]^{m}\right)^{*} \otimes \mathcal{B}$ is surjective, by right exactness of $-\otimes_{\mathbf{Z}[G]} \mathcal{B}$. From the correspondence

$$
H^{3}(X(\alpha) ; \mathcal{B}) \longleftrightarrow\left(\mathbf{Z}[G]^{M}\right)^{*} \otimes \mathcal{B} / \operatorname{Im}\left((i \circ \alpha)^{*} \otimes 1_{\mathcal{B}}\right)
$$

we see that $H^{3}(X(\alpha) ; \mathcal{B})=0$ for all local coefficient systems $\mathcal{B}$.

Observe that if $\mathbf{E}$ is any algebraic 2-complex, there is a natural chain map $\psi: \mathcal{S}(\mathbf{E}) \rightarrow \mathbf{E}$ which is the projection on the top two terms, but is otherwise the identity. The following is an obvious consequence of Browning's Stability Theorem $[2],[11]$.

Proposition 6.10. If $\mathbf{E}_{1}, \mathbf{E}_{2}$ are algebraic 2-complexes over $\mathbf{Z}[G]$ then

$$
\mathcal{S}^{n}\left(\mathbf{E}_{1}\right) \simeq \mathcal{S}^{m}\left(\mathbf{E}_{2}\right)
$$

for some $n, m \geq 1$.

Corresponding to any algebraic 2-complex over $\mathbf{Z}[G]$, we can now construct a three dimensional cell complex which is cohomologically 2-dimensional.

Theorem 6.11. Let $\mathbf{E}$ be an algebraic 2-complex over the finite group $G$; then there exists a finite 3-dimensional cell complex $X$ such that

(i) $\pi_{1}(X) \cong G$;

(ii) $H^{3}(X ; \mathcal{B})=0$ for all local coefficient systems $\mathcal{B}$; and

(iii) $\langle X\rangle$ is homotopy equivalent to $\mathbf{E}$.

Proof. Write $\mathbf{E}$ thus

$$
\mathbf{E}=\left(0 \rightarrow J \rightarrow \mathbf{Z}[G]^{\gamma} \stackrel{\delta_{2}}{\rightarrow} \mathbf{Z}[G]^{\beta} \stackrel{\delta_{1}}{\rightarrow} \mathbf{Z}[G] \stackrel{\epsilon}{\rightarrow} \mathbf{Z} \rightarrow 0\right) .
$$

Let $L$ be a finite 2 -complex with $\pi_{1}(L) \cong G$; then by $(6.10)$,

$$
\mathcal{S}^{n}(\mathbf{E}) \simeq \mathcal{S}^{m}(\mathcal{C}(L))
$$

for some $n, m \geq 1$. However, $\mathcal{S}^{m}(\mathcal{C}(L)) \cong \mathcal{C}\left(L \vee m S^{2}\right)$. Put $K=L \vee m S^{2}$, and let $\varphi: \mathcal{C}(K) \rightarrow \mathcal{S}^{n}(\mathbf{E})$ be a weak homotopy equivalence. In particular, $\varphi$ induces an isomorphism

$$
\varphi: \pi_{2}(K) \stackrel{\simeq}{\rightarrow} J \oplus \mathbf{Z}[G]^{n} .
$$

If $\psi: \mathcal{S}^{n}(\mathbf{E}) \rightarrow \mathbf{E}$ is the natural chain projection, then $\psi \circ \varphi: \mathcal{C}(K) \rightarrow \mathbf{E}$ has the property that $\operatorname{Ker}\left(\psi \circ \varphi: \pi_{2}(K) \rightarrow J\right)$ is isomorphic to $\mathbf{Z}[G]^{n}$. Choose a $\mathbf{Z}[G]$ basis $\alpha_{1}, \alpha_{2}, \cdots, \alpha_{m}$ for $\operatorname{Ker}(f)$, and form $X(\alpha)$ by attaching 3 -cells $E_{1}^{(3)}, E_{2}^{(3)}, \cdots, E_{m}^{(3)}$ to $K$ by means of $\alpha_{1}, \alpha_{2}, \cdots, \alpha_{m}$; that is,

$$
X(\alpha)=K \cup_{\alpha_{1}} E_{1}^{(3)} \cup_{\alpha_{2}} E_{2}^{(3)} \cdots \cdots \cup_{\alpha_{m}} E_{m}^{(3)} .
$$

It follows from $(6.9)$ that $H^{r}(X(\alpha) ; \mathcal{B})=0$ for all $r \geq 3$, and it is straightforward to see that $\langle X\rangle$ is homotopy equivalent to $\mathbf{E}$. 
From (6.7) and (6.11) we obtain:

Realization Criterion. The $\mathrm{D}(2)$ property holds for the finite group $G$ if and only if every algebraic 2-complex over $\mathbf{Z}[G]$ is geometrically realized.

\section{References}

[1] R. F. Beyl, M. P. Latiolais and N. Waller, Classification of 2-complexes whose finite fundamental group is that of a 3-manifold, Proc. Edinburgh Math. Soc. 40 (1997), 69-84.

[2] W. Browning, Homotopy types of certain finite C. W. complexes with finite fundamental group, Ph. D. Thesis, Cornell University, 1978.

[3] W. Browning, Truncated projective resolutions over a finite group (unpublished notes), ETH, April 1979.

[4] W. Browning, Finite CW complexes of cohomological dimension two with finite abelian fundamental group (unpublished notes), ETH, May 1979.

[5] J. F. Carlson, Modules and group algebras, Lecture Notes in Mathematics, ETH, Birkhäuser, 1996.

[6] W. H. Cockroft and R. G. Swan, On the homotopy types of certain two-dimensional complexes, Proc. London Math. Soc. (3) 11 (1961), 194-202.

[7] M. N. Dyer and A. J. Sieradski, Trees of homotopy types of two-dimensional CW complexes, Comment. Math. Helv. 48 (1973), 31-44.

[8] M. N. Dyer and A. J. Sieradski, Trees of homotopy types of two-dimensional CW complexes II, Trans. Amer. Math. Soc. 205 (1975), 115-125.

[9] R. H. Fox, Free differential calculus V, Ann. of Math. 71 (1960), 408-422.

[10] K. W. Gruenberg, Homotopy classes of truncated projective resolutions, Comment. Math. Helv. 68 (1993), 579-598.

[11] M. Gutierrez and M. P. Latiolais, Partial homotopy type of finite two-complexes, Math. Zeit. 207 (1991) 359-378.

[12] H. Jacobinski, Genera and decompositions of lattices over orders, Acta Math. 121 (1968), $1-29$.

[13] F. E. A. Johnson, The derived category of an involuted algebra, Preprint, University College London, 1997.

[14] F. E. A. Johnson, Relative Yoneda Theory, Preprint, University College London, 1999.

[15] F. E. A. Johnson, Stable modules and the structure of Poincaré 3-complexes, in: Geometry and Topology Aarhus, Proceedings of the $6^{\text {th }}$ Aarhus Topology Conference, 227-248, A.M.S. Contemporary Mathematics, 258, 2000.

[16] F. E. A. Johnson, Explicit homotopy equivalences in dimension two, Math. Proc. Camb. Phil. Soc. 133 (2002), 411-430.

[17] F. E. A. Johnson, Counting homotopy types in dimension two, Preprint, University College London, 2001.

[18] S. Mac Lane, Homology, Springer-Verlag, 1963.

[19] S. Mac Lane and J. H. C. Whitehead, On the 3-type of a complex, Proc. Nat. Acad. Sci. 36 (1950), 41-48.

[20] W. Metzler, Über den Homotopietyp zweidimensionaler CW-komplexe und Elementartransformationen bei Darstellungen von Gruppen durch Erzeugenden und definierende Relationen, J. Reine Angew. Math. 285 (1976), 7-23.

[21] E. H. Spanier, Algebraic Topology, McGraw Hill, 1967.

[22] R. G. Swan, Periodic resolutions for finite groups, Ann. of Math. 72 (1960), 267-291.

[23] R. G. Swan, The Grothendieck ring of a finite group, Topology 2 (1963), 85-110.

[24] R. G. Swan, Minimal resolutions for finite groups, Topology 4 (1965), 193-208 
[25] R. G. Swan, K-Theory of finite groups and orders (notes by E. G. Evans), Lecture Notes in Mathematics 149, Springer-Verlag, 1970.

[26] R. G. Swan, Projective modules over binary polyhedral groups, Journal für die Reine und Angewandte Mathematik 342 (1983), 66-172.

[27] C. B. Thomas, A reduction theorem for free actions by the group $Q(8 n, k, l)$ on $S^{3}, B u l l$. London Math. Soc. 20 (1988), 65-67.

[28] H. Tietze, Über die topologischen Invarianten mehrdimensionaler Mannigfaltigkeiten, Monatsh. Math. Phys. 19 (1908), 1-118.

[29] C. T. C. Wall, Finiteness conditions for CW Complexes, Ann. of Math. 81 (1965), 56-69.

[30] C. T. C. Wall, Poincaré complexes I, Ann. of Math. 86 (1967), 213-245.

[31] J. H. C. Whitehead, Combinatorial homotopy II, Bull. Amer. Math. Soc. 55 (1949), 456496.

[32] N. Yoneda, On the homology theory of modules: J. Fac. Sci. Tokyo, Sec. I 7 (1954), $193-227$.

F. E. A. Johnson

Department of Mathematics

University College London

Gower Street

London WC1E 6BT

U.K.

e-mail: feaj@math.ucl.ac.uk

(Received: January 19, 2001)

To access this journal online:

(4) http://www.birkhauser.ch 